\title{
The Arabidopsis thaliana double-stranded RNA binding protein DRB1 directs guide strand selection from microRNA duplexes
}

\author{
ANDREW L. EAMENS, ${ }^{1,2}$ NEIL A. SMITH, ${ }^{1}$ SHAUN J. CURTIN, ${ }^{1,4}$ MING-BO WANG, ${ }^{1}$ \\ and PETER M. WATERHOUSE ${ }^{1,2,3}$ \\ ${ }^{1}$ Commonwealth Scientific and Industrial Research Organisation Plant Industry, Canberra, Australian Capital Territory 2601, Australia \\ ${ }^{2}$ School of Molecular and Microbial Bioscience, University of Sydney, Sydney, New South Wales 2006, Australia \\ ${ }^{3}$ School of Biological Sciences, University of Sydney, Sydney, New South Wales 2006, Australia
}

\begin{abstract}
In Arabidopsis thaliana (Arabidopsis), DICER-LIKE1 (DCL1) functions together with the double-stranded RNA binding protein (dsRBP), DRB1, to process microRNAs (miRNAs) from their precursor transcripts prior to their transfer to the RNA-induced silencing complex (RISC). miRNA-loaded RISC directs RNA silencing of cognate mRNAs via ARGONAUTE1 (AGO1)-catalyzed cleavage. Short interefering RNAs (siRNAs) are processed from viral-derived or transgene-encoded molecules of doublestranded RNA (dsRNA) by the DCL/dsRBP partnership, DCL4/DRB4, and are also loaded to AGO1-catalyzed RISC for cleavage of complementary mRNAs. Here, we use an artificial miRNA (amiRNA) technology, transiently expressed in Nicotiana benthamiana, to produce a series of amiRNA duplexes with differing intermolecular thermostabilities at the $5^{\prime}$ end of duplex strands. Analyses of amiRNA duplex strand accumulation and target transcript expression revealed that strand selection (amiRNA and amiRNA*) is directed by asymmetric thermostability of the duplex termini. The duplex strand possessing a lower $5^{\prime}$ thermostability was preferentially retained by RISC to guide mRNA cleavage of the corresponding target transgene. In addition, analysis of endogenous miRNA duplex strand accumulation in Arabidopsis drb1 and drb2345 mutant plants revealed that DRB1 dictates strand selection, presumably by directional loading of the miRNA duplex onto RISC for passenger strand degradation. Bioinformatic and Northern blot analyses of DCL4/DRB4-dependent small RNAs (miRNAs and siRNAs) revealed that small RNAs produced by this DCL/dsRBP combination do not conform to the same terminal thermostability rules as those governing DCL1/DRB1-processed miRNAs. This suggests that small RNA processing in the DCL1/DRB1-directed miRNA and DCL4/DRB4-directed sRNA biogenesis pathways operates via different mechanisms.
\end{abstract}

Keywords: miRNA; siRNA; terminal thermostability; duplex strand selection; DRB1; DCL1; DRB4; DCL4; AGO1; RNA silencing

\section{INTRODUCTION}

In eukaryotes, numerous biological processes that are crucial for normal development, including the formation of heterochromatin and protection against invading nucleic acids derived from viruses or replicating transposons, are regulated by RNA silencing mechanisms (Rand et al. 2005; Tagami et al. 2007). RNA silencing results in the sequencespecific inhibition of gene expression at either the transcriptional or post-transcriptional level and is guided by

\footnotetext{
${ }^{4}$ Present address: Department of Agronomy and Plant Genetics, University of Minnesota, 1991 Upper Buford Circle, St. Paul, MN, 55108, USA.

Reprint requests to: Peter M. Waterhouse, School of Biological Sciences, University of Sydney, Sydney, New South Wales 2006, Australia; e-mail: peter.waterhouse@usyd.edu.au; fax: 61-02-91140988.

Article published online ahead of print. Article and publication date are at http://www.rnajournal.org/cgi/doi/10.1261/rna.1646909.
}

21-24-nucleotide (nt) small RNAs (sRNAs) generated from longer double-stranded RNA (dsRNA) molecules by the RNase III-like endonuclease Dicer (Gregory et al. 2005; Brodersen and Vionnet 2006). Dicer (Dcr) functions in concert with proteins encoded by two other gene families, the dsRNA binding (dsRBP) and Argonaute (AGO) gene families, to form a mature sRNA-loaded RNA-induced silencing complex (RISC), the catalytic center that executes a specific RNA silencing mechanism (Rand et al. 2005; Liu et al. 2007). Small RNAs functioning at the post-transcriptional level are classified into two distinct categories: smallinterfering RNAs (siRNAs) and microRNAs (miRNAs), based on their mode of biogenesis (Mallory and Vaucheret 2006). Dicer cleavage of long perfectly dsRNA derived from the transcription of repetitive DNA sequences, transposons, viruses, or natural antisense gene pairs, or from introduced transgene-encoded hairpins, results in the biogenesis of 
siRNAs (Schwarz et al. 2003; Pontier et al. 2005). In contrast, miRNAs result from Dcr processing of endogenous noncoding mRNA precursor transcripts that have the ability to fold back onto themselves to form stem-loop structures of imperfectly dsRNA (Nakano et al. 2006).

In Drosophila melanogaster, for example, the dsRBP R2D2 interacts with Dcr-2 to form the RISC-loading complex (RLC) during siRNA biogenesis. The RLC heterodimer asymmetrically loads siRNA duplexes to the AGO family member Ago2, with R2D2 binding to the more stable end of the siRNA duplex and Dcr-2 binding to the other end so as to orientate the passenger strand for Ago2catalyzed cleavage. The remaining intact strand of the siRNA duplex, the guide strand, then pairs with complementary mRNAs to specially guide target cleavage via the Slicer activity of Ago2 (Tomari et al. 2004; Matranga et al. 2005; Rand et al. 2005). MicroRNA biogenesis on the other hand, requires two unique RNase III endonuclease/dsRBP combinations to efficiently process the precursor molecules of imperfectly dsRNA. In the nucleus, primary-miRNA (pri-miRNA) transcripts are processed into smaller, $\sim 60$ 70-nt precursor-miRNAs (pre-miRNAs) by the RNase IIIlike endonuclease Drosha (Gregory et al. 2005; Song et al. 2007). Drosha requires the dsRBP Pasha to catalyze this initial cleavage step at the base of the pre-miRNA stemloop structure (Kurihara et al. 2006). These smaller hairpinshaped intermediates are then exported to the cytoplasm in an Exportin-5 (Exp-5)-dependent process (Li et al. 2003). In the cytoplasm, Dcr-1 performs the second cleavage step of the miRNA biogenesis pathway to liberate the miRNA/ miRNA $^{\star}$ duplex from the pre-miRNA stem-loop structure (Kurihara and Watanabe 2004). Like the initial cleavage step, efficient Dcr-1-mediated pre-miRNA processing requires the coordinated action of R3D1/Loquacious (Loqs), a cytoplasmically localized dsRBP (Liu et al. 2007). One strand of the duplex, the mature miRNA, is then incorporated into RISC to guide RNA silencing of target genes, and, in insects and animals, the predominant mode of miRNA-directed silencing is translation inhibition (Bagga et al. 2005).

In plants, however, the predominant mode of RNA silencing mediated by miRNA-loaded RISC is mRNA cleavage (Baumberger and Baulcombe 2005; Tagami et al. 2007). The steps involved in the biogenesis of plant miRNAs also differ from, and are not as well understood as, those of the miRNA maturation pathway in animals, and the identification of factors involved in plant miRNA biogenesis stem from the characterization of phenotypic mutants originally isolated in developmental screens and subsequently shown to be partially defective in miRNA accumulation (Vaucheret et al. 2004). For example, of the four DICER-LIKE (DCL) genes encoded by the Arabidopsis genome (Margis et al. 2006), miRNA accumulation has been shown to almost exclusively depend on the action of DCL1 (Park et al. 2002; Reinhart et al. 2002; Bouché et al.
2006). The nuclear-localized DCL1 has both Drosha and Dicer activities, executing both cleavage steps to liberate the miRNA duplex from pri- and pre-miRNA intermediates (Kurihara and Watanabe 2004). In addition, and as shown for Drosha- and Dicer-directed dsRNA cleavage in Drosophila, DCL1 interacts with, and requires the combined action of, the dsRBP, HYPONASTIC LEAVES1 (HYL1; referred to as DRB1 from here on), to efficiently process miRNAs from their precursor transcripts (Han et al. 2004; Vazquez et al. 2004). The accuracy of pri- to pre-miRNA processing by DCL1/DRB1 has been shown to also rely on SERRATE (SE), a $\mathrm{C}_{2} \mathrm{H}_{2}$ zinc-finger protein unique to plants (Yang et al. 2006; Fujioka et al. 2007; Dong et al. 2008). The 3 ' dinucleotide overhangs of miRNA duplexes are methylated by the sRNA-specific methyltransferase HUA ENHANCER1 (HEN1). HEN1 has also been shown to methylate siRNA duplexes, a modification that is thought to protect all sRNA species from subsequent degradation (Boutet et al. 2003; Kurihara et al. 2006). HASTY (HST), the Arabidopsis Exp-5 ortholog, appears to play a role in the nuclear export of methylated miRNA duplexes; however, its exact role in plant miRNA biogenesis remains unclear, as several species of miRNA are exported to the cytoplasm by a HSTindependent mechanism (Park et al. 2005). In the cytoplasm, AGO1-catalyzed RISC uses the mature miRNA as a guide for Slicer-directed cleavage of homologous mRNAs to repress gene expression (Baumberger and Baulcombe 2005).

As shown for DCL1, DCL4 requires the dsRBP DRB4 for the phased production of trans-acting siRNAs (tasiRNAs), an endogenous class of plant-specific sRNA (Adenot et al. 2006). In tasiRNA biogenesis, DCL1/DRB1/AGO-generated miRNAs—namely, miR171 (AGO1) and miR390 (AGO7) cleave noncoding mRNAs transcribed from TAS loci (Allen et al. 2005). This miRNA-directed cleavage event identifies the TAS transcript as a template for dsRNA synthesis by RNA-DEPENDENT RNA POLYMERASE6 (RDR6) with the help of the coiled-coil protein, SUPPRESSOR OF GENE SILENCING3 (SGS3) (Adenot et al. 2006). The dsRNA molecule is then processed in a phased manner, initiating at the miRNA cleavage site by DCL4/DRB4 to produce sequential 21-nt tasiRNAs that are loaded into AGO1 to direct cleavage of cognate mRNAs in trans (Gasciolli et al. 2005; Xie et al. 2005). In addition to tasiRNA biogenesis, the concerted action of DCL4/DRB4 is also required for the efficient production of the 21-nt size class of exogenous siRNAs derived from either replicating viruses or from an introduced hairpin RNA (hpRNA)encoding transgene (Bouché et al. 2006; Curtin et al. 2008).

The similarities between the RNA silencing machineries producing sRNAs in Drosophila and Arabidopsis led us to investigate whether DRB1 and DRB4 function in a similar manner to the Drosophila dsRBPs, Loqs and R2D2, in the Arabidopsis miRNA and siRNA biogenesis pathways, respectively. 


\section{RESULTS}

\section{Bioinformatic survey of our sRNA sequencing data}

Rajagopalan et al. (2006) have previously shown that for the majority of miRNAs identified in their Arabidopsis sequencing population, one strand of the miRNA/miRNA* duplex, the miRNA guide strand, was preferentially selected for RISC incorporation. They went on to suggest that the preferential selection of the miRNA over its miRNA* counterpart was due to this duplex strand expressing a more favorable thermodynamic stability at its $5^{\prime}$ terminus. We were therefore interested to see if we could identify the same trend in our own sRNA sequencing set. The miRNA-specific sRNA sequencing data were produced as part of our analysis of the siRNA population generated following the introduction of a transgene hpRNA vector into the Arabidopsis genome. Overall, 58 unique miRNA or miRNA* sequences (with a combined miRNA/miRNA* read score greater than 5) were identified and grouped into six classes depending on (1) the thermodynamic stability of the duplex from which the miRNA or miRNA* was processed, and (2) whether the selected duplex strand was influenced by the thermodynamic properties of the duplex. The six miRNA duplex classes and their associated miRNA/miRNA* sequencing reads are presented in Table 1, and a complete summary of the miRNAspecific sRNA sequencing set is provided in Supplemental Data 1.

The majority of miRNAs are processed from Class I duplexes, accounting for 41 of 58 miRNAs identified, or $96.1 \%$ of the miRNA-specific sRNA sequencing reads. These Class I duplexes are thermodynamically asymmetrical, with one duplex strand having a lower thermostability at its $5^{\prime}$ terminus, compared with a stronger dsRNA base-pairing at position 19 of the same duplex strand (corresponding to position 1 of the miRNA* strand). Class II duplexes are also asymmetrical; however, the miRNA strand is either weakly or not selected over the miRNA* strand. Class III and IV miRNA duplexes are also thermodynamically asymmetrical. Class III duplexes have a lower thermostability at the $5^{\prime}$ end of the miRNA* strand, with the miRNA* preferentially selected over the opposite duplex strand. Class IV duplexes also express a lower thermostability at the $5^{\prime}$ end of the miRNA* duplex strand; however, the miRNA is still preferentially selected over the miRNA* strand from this class of duplex. Class V and VI miRNA duplexes are symmetrical, with equivalent thermostability at the $5^{\prime}$ terminus of both duplex strands. The thermodynamic symmetry of Class $\mathrm{V}$ duplexes resulted in equal processing of the miRNA and miRNA* strands from this class of miRNA duplex. However, for Class VI duplexes, the miRNA guide strand was favored for accumulation despite the symmetry of the duplex.

Overall, the sequence analysis showed a strong correlation between the preferential selection of plant miRNAs over their partner miRNA* sequences and differential thermostability of duplex termini. The three miRNA duplex classes that are strongly influenced by the thermostability properties of the selected duplex strand (Classes I, III, and V) accounted for $96.5 \%$ of the miRNA-specific sRNA sequencing population, suggesting that duplex terminal thermostability, or dsRNA base-pairing at duplex strand positions 1 and 19, is highly influential in determining which duplex strand is selected for RISC incorporation. However, miRNA classes II, IV, and VI are counter to this correlation. Therefore, we decided to directly test the effects of differential thermostability of duplex termini on their preferential selection and incorporation into RISC to guide RNA silencing using artificial miRNA (amiRNA) technology.

TABLE 1. Influence of miRNA duplex symmetry on strand selection

\begin{tabular}{|c|c|c|c|c|c|c|}
\hline $\begin{array}{l}\text { Duplex } \\
\text { class }\end{array}$ & & $\begin{array}{c}\text { Appropriate } \\
\text { duplex strand selected }\end{array}$ & $\begin{array}{l}\text { Class } \\
\text { members }\end{array}$ & $\begin{array}{l}\text { miRNA } \\
\text { reads }\end{array}$ & $\begin{array}{l}\text { miRNA* } \\
\text { reads }\end{array}$ & $\%$ Sequencing population \\
\hline \multicolumn{7}{|c|}{ Duplex description } \\
\hline $\mathrm{I}^{\mathrm{a}}$ & Asymmetrical duplex-miRNA strand bias & Yes & 41 & $99.3 \%$ & $0.7 \%$ & 96.1 \\
\hline${ } \mathrm{I}^{\mathrm{b}}$ & Asymmetrical duplex-miRNA strand bias & No & 4 & $74.3 \%$ & $25.7 \%$ & 3.0 \\
\hline$\| I^{\mathrm{a}}$ & Asymmetrical duplex-miRNA* strand bias & Yes & 2 & $16.7 \%$ & $83.3 \%$ & 0.2 \\
\hline $\mathrm{IV}^{\mathrm{b}, \mathrm{c}}$ & Asymmetrical duplex-miRNA* strand bias & No & 2 & $94.2 \%$ & $5.8 \%$ & 0.5 \\
\hline $\mathrm{V}^{\mathrm{a}}$ & Symmetrical duplex-no strand bias & Yes & 5 & $68.2 \%$ & $31.8 \%$ & 0.1 \\
\hline $\mathrm{Vl}^{\mathrm{b}, \mathrm{c}}$ & Symmetrical duplex-no strand bias & No & 4 & $97.4 \%$ & $2.6 \%$ & 0.1 \\
\hline \multicolumn{7}{|c|}{ Influence of symmetry of miRNA duplex strand selection } \\
\hline $\mathrm{I}, \mathrm{III}, \mathrm{V}^{\mathrm{a}}$ & Duplex strand selection influenced & - & 48 & 349,852 & 3199 & 96.5 \\
\hline $\mathrm{II}, \mathrm{IV}, \mathrm{VI} \mathrm{b}^{\mathrm{b}}$ & Duplex strand selection not influenced & - & 10 & 10,011 & 2933 & 3.5 \\
\hline $\mathrm{I}-\mathrm{VI}$ & Total number of miRNAs identified & - & 58 & 359,863 & 6132 & 100.0 \\
\hline
\end{tabular}

${ }^{a}$ miRNA or miRNA* strand selection influenced by duplex thermodynamic symmetry.

bmiRNA or miRNA* strand selection not influenced by duplex thermodynamic symmetry.

${ }^{C}$ miRNA strand selected over miRNA* strand irrespective of thermodynamic duplex symmetry. 


\section{Strand choice from amiRNA duplexes}

More recently, it has been shown that several of the AGO proteins encoded by the Arabidopsis genome preferentially associate with sRNAs expressing a specific $5^{\prime}$ terminal nucleotide (Mi et al. 2008; Takeda et al. 2008), suggesting that $5^{\prime}$ terminal stability may not be the only determinant of miRNA or siRNA duplex strand selection for RISC incorporation. To assess the influence of both $5^{\prime}$ terminal stability and $5^{\prime}$ nucleotide composition on miRNA strand selection, we developed a series of strand choice (SC) amiRNA duplexes and transiently expressed each duplex in Nicotiana benthamiana leaves along with either the sense or antisense version of the target transgene, $\beta$-glucuronidase (GUS). The nine amiRNA duplexes shown in Figure 2 (see below) were incorporated into our amiRNA plant expression vector pBlueGreen (Fig. 1A) and transformed into Agrobacterium tumefaciens. Cultures of each amiRNA duplex were mixed with an Agrobacterium culture expressing either the sense or antisense mRNA of the GUS target transgene (Fig. 1B) and coinfiltrated into $N$. benthamiana leaves.

The amiRNA duplexes, SC-1 and SC-2 (Fig. 2A,B), are asymmetrical due to the presence of a $\mathrm{G}: \mathrm{U}$ wobble pair at position 1 of the antisense (sensemRNA-targeting [blue]) and sense (antisense-mRNA-targeting [red]) strand of amiRNA duplexes SC-1 and SC-2, respectively. The G:U wobble pair of SC-1 was replaced with a $\mathrm{U}-\mathrm{U}$ mismatch to create amiRNA duplex SC-3 (Fig. 2C). This amiRNA duplex maintains the asymmetrical thermostability of SC-1, but made both duplex strands express a uracil residue at their $5^{\prime}$ terminus. The asymmetry of these three duplexes resulted in the biased silencing of one target transgene at the expense of the other. Strand choice amiRNA vectors SC-1 and SC-3 are thermodynamically less stable at the $5^{\prime}$ end of their duplex's antisense strand and gave highly efficient silencing of the GUS sense transgene, but very inefficient silencing of the GUS antisense transgene (Fig. 2A,C). Conversely, moving the

B

C

D of GFP.
G:U wobble pairing at position 1 of the antisense strand in amiRNA duplex SC-1 to position 1 of the sense strand in SC-2 greatly increased its silencing of the GUS antisense transgene, and almost completely abolished its silencing of
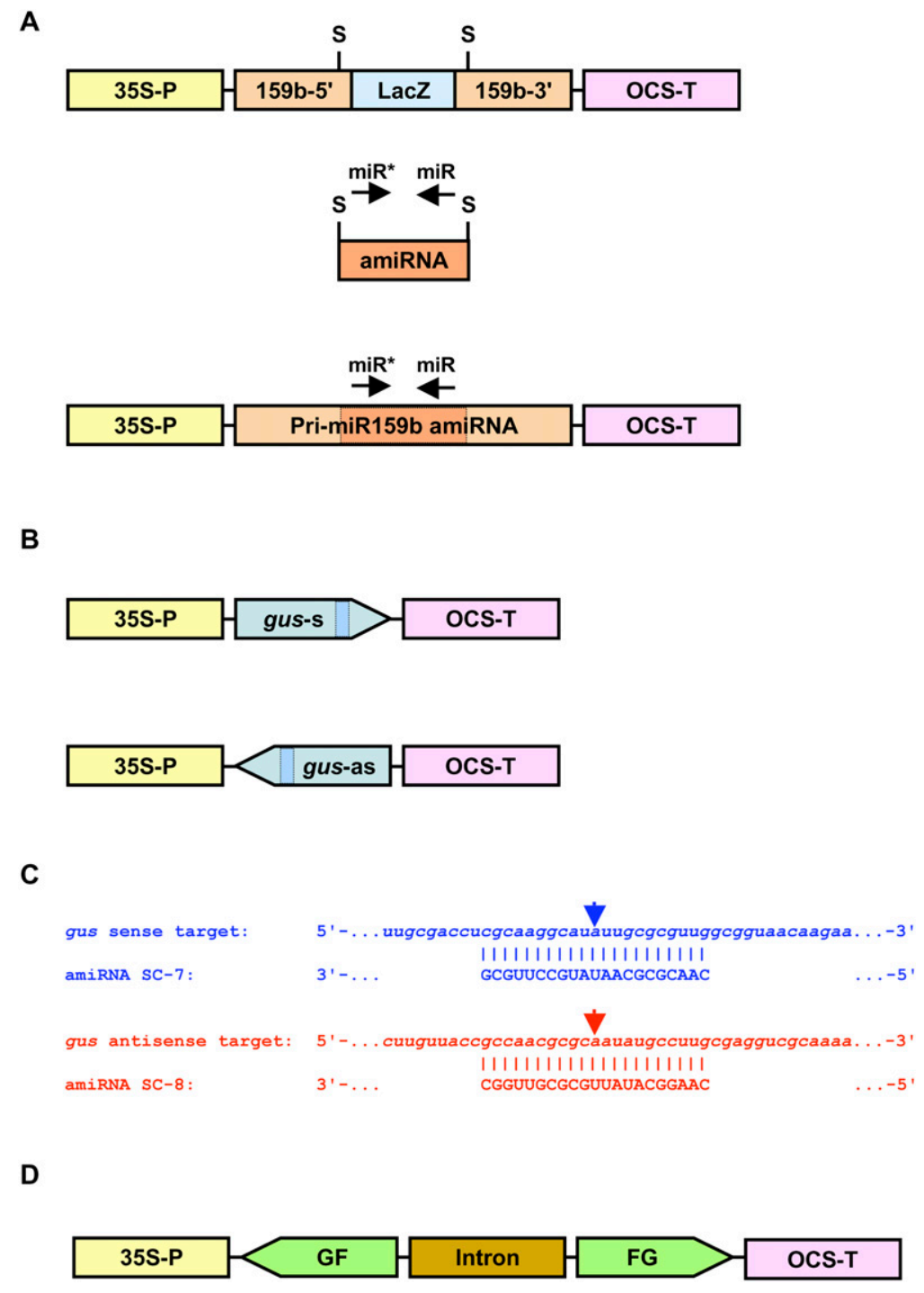

FIGURE 1. Vectors used in this study. (A) Schematic representation of the amiRNA plant expression vector pBlueGreen. The endogenous MIR159b precursor transcript sequence (premiRNA) was replaced with the LacZ gene (blue box) to separate $5^{\prime}$ and $3^{\prime}$ arms of the MIR159b primary transcript (light orange boxes). PCR product of the MIR159b precursor transcript (dark orange box) containing amiRNA guide and passenger strand sequences and flanked by SapI restriction sites (S) was amplified and cloned into the pri-miRNA sequence (replacing the LacZ gene) using the corresponding SapI restriction sequences of the pBlueGreen vector to produce the amiRNA plant expression vectors. (B) Schematic representation of the GUS sense (pART27:GUS-S) and GUS antisense (pART27:GUS-AS) plant expression vectors. The GUS transgene, either in the sense or antisense orientation (light blue boxes), was constitutively expressed by the Cauliflower Mosaic Virus 35S promoter (35S-P; yellow boxes). (Dark blue, dashed-line box) Position of the recognition site of the transgenes targeted by the series of amiRNA strand choice vectors. $(C)$ The RNA sequences of the GUS sense and antisense target transgene flanking the strand choice amiRNA cleavage site (arrows). The perfectively matched sequences of mature amiRNA processed from amiRNA strand choice vectors SC-7 and SC-8 are given below their respective mRNA targets. $(D)$ Hairpin RNA transgene of the GF portion 


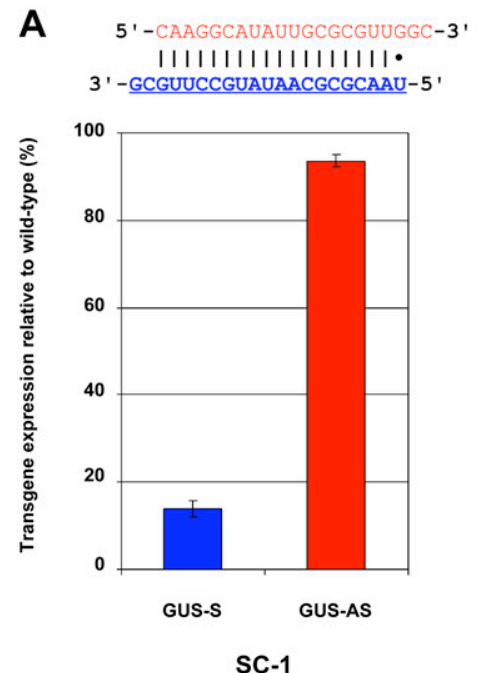

D 5'-CaAggcauaudgcgcguUugc-3 ' |||||||||||||||||| $\mid$ 3 ' -GCGUUCCGUAUAACGCGCAAA- 5 '

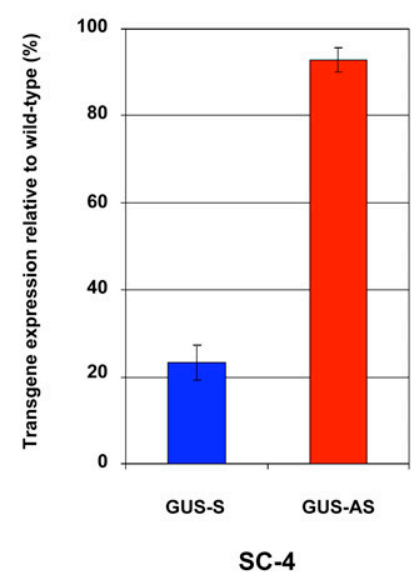

G $5^{\prime}-$-CAAGgCAUAUUGCGCGUUAGC-3'
$111111111111111111 \times$
$3^{\prime}-$-GCGUUCCGUAUAACGCGCAAC-5

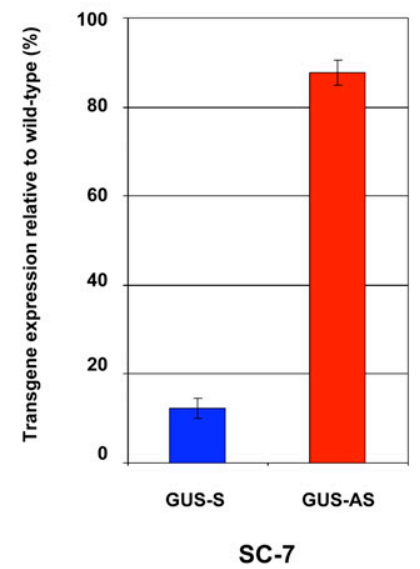

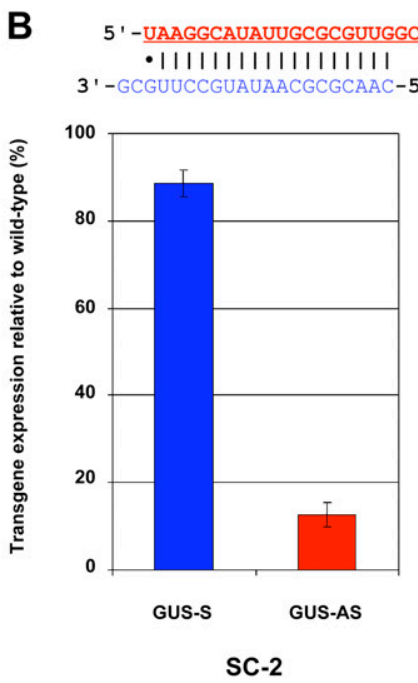

E
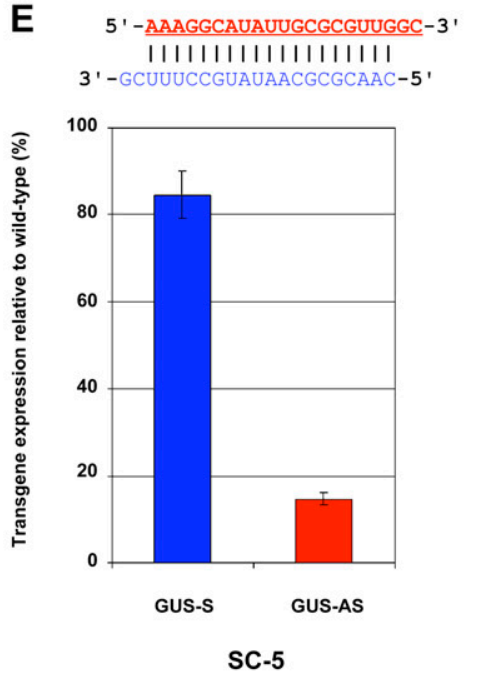

H
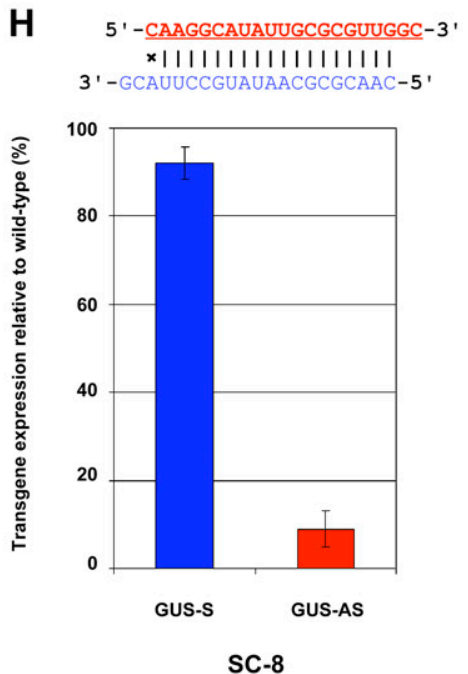

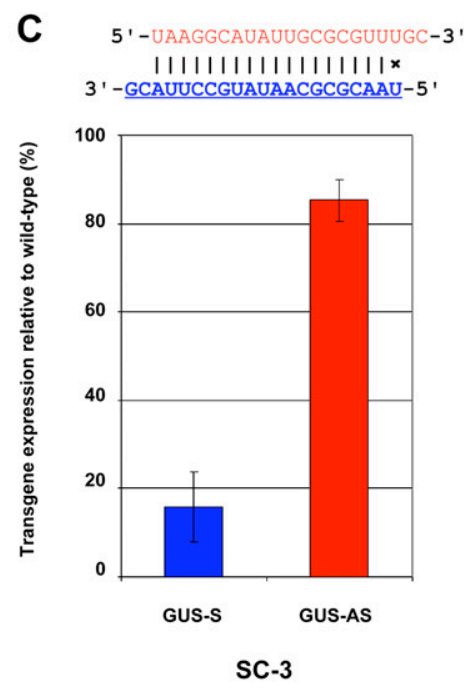
$\mathbf{F}$ 5' -AAAGGCAUAUUGCGCGUUUGC-3' 1111111111111111111 3 ' - GCUUUCCGUAUAACGCGCAAA-5 '

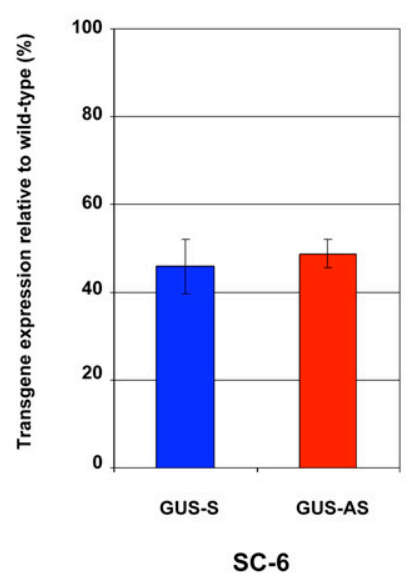

I

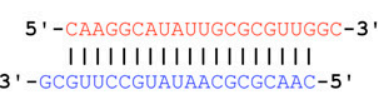

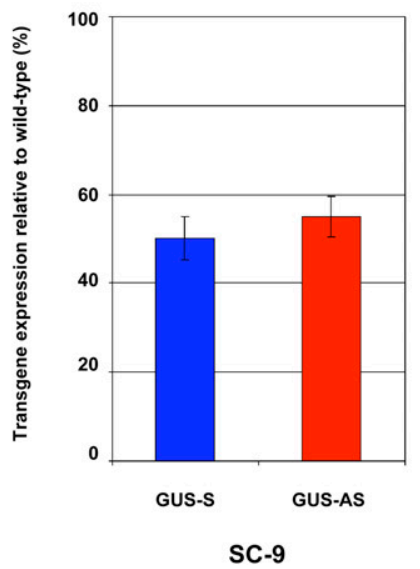

FIGURE 2. Target transgene expression in GUS strand choice amiRNA transformants. $(A-I)$ Expression of the GUS sense (blue colored columns) and GUS antisense (red colored columns) target transgenes in amiRNA transformant lines. (Bolded, underlined sequence in $A-E, G, H$ ) Preferentially selected duplex strand from each of the seven asymmetrical amiRNA strand choice vectors analyzed in this study. Each sample was normalized to the respective selectable marker genes of the amiRNA vector (Basta) and the target transgene vector (kanamycin). The expression of all analyzed transcripts was also normalized to the Arabidopsis gene Cyclophilin (At2g29960). The relative expression level of each target transgene was then compared with the expression levels of controls (GUS sense or antisense target transgene alone) to determine the silencing efficiency of each GUS strand choice amiRNA duplex. Error bars represent the standard error of the mean (SEM) between three biological replicates. 
the GUS sense transgene (Fig. 2B). Northern blot hybridization analyses of sense and antisense strand accumulation from amiRNA duplexes SC-1 and SC-2 (Fig. 3A,B) demonstrated that the silencing polarity of these two vectors was directly correlated with high accumulation levels of either the amiRNA-antisense (blue) or amiRNA*-sense (red) duplex strand, respectively. Furthermore, the sRNA accumulation profile (Fig. 3) and asymmetric silencing (Fig. 2C) by amiRNA duplex SC-3 demonstrates that this was directed by the asymmetric terminal thermostabilities of duplex strands rather than the identity of the $5^{\prime}$ terminal nucleotide. In all three cases, the degree of GUS sense or antisense transgene silencing was directly correlated with the abundance of the complementary amiRNA duplex strand (Fig. 3).

A different strategy was used to alter the $5^{\prime}$ stability of either the sense or antisense strand in the asymmetric amiRNA duplexes SC-4 and SC-5. The sequence of SC-4 is identical to that of SC-1 (Fig. 2A), except that the G:U wobble pair at the $5^{\prime}$ terminus of the antisense strand was replaced with an A:U pairing (Fig. 2D). This alteration in SC-4, which maintains the $5^{\prime}$ asymmetry present in SC- 1 , enabled efficient silencing of the GUS sense transgene and gave a concomitant lack of silencing of the antisense target transgene (Fig. 2D). Interchanging the terminal stabilities of SC-4 by swapping the A:U and G:C base-pairings at positions 1 and 19 of the antisense strand to the corresponding

A

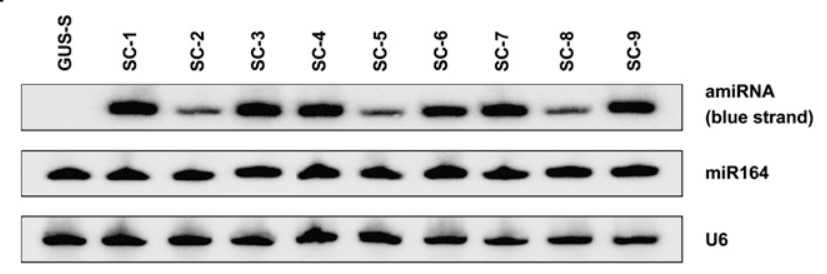

B



FIGURE 3. Small RNA accumulation in GUS strand choice amiRNA transformant lines. (A) Small RNA accumulation (amiRNA duplex strand) in amiRNA transformant lines infiltrated with the GUS sense target transgene alone and in combination with the nine amiRNA duplexes, SC-1-SC-9. Total RNA was probed with U6 (loading control), miR164 (internal control), and an amiRNA duplex antisense $(-)$ strand-specific DNA oligonucleotide. (B) Small RNA accumulation (amiRNA* duplex strand) in amiRNA transformant lines infiltrated with the GUS antisense target transgene alone and in combination with the nine amiRNA duplexes, SC-1-SC-9. Total RNA was probed with U6 (loading control), miR164 (internal control), and an amiRNA duplex sense (+) strand-specific DNA oligonucleotide. positions of the duplex sense strand to generate SC-5 reversed the silencing polarity (Fig. 2E), mirroring the effects of changing the thermodynamic stabilities of SC-1SC-2. Furthermore, and as shown for amiRNA duplexes SC-1 and SC-2, Northern blot hybridization analyses clearly indicated that the changes to the silencing efficiencies associated with amiRNA duplexes SC- 4 and SC- 5 were directly attributable to the accumulation of strand-specific sRNAs (Fig. 3). In contrast to amiRNA duplexes SC-1 through to SC-5, the SC-6 duplex is symmetrical, with both duplex strands starting with an A:U dsRNA base-pair. This thermodynamic symmetry of amiRNA duplex SC-6 led to comparable levels of silencing of both target transgenes (Fig. 2F), and Northern blot analyses showed no preferential selection for either strand of this symmetrical duplex (Fig. 3). Comparison of the accumulation profiles of the sRNA species processed from amiRNA duplexes SC-1-SC-6 (Fig. 3) suggested that the suppression of silencing in these transformant lines was not a result of the introduction of a single nucleotide mismatch between the sense or antisense amiRNA sRNA and their respective target mRNAs (Fig. 1C). If the introduced mismatch was interfering with the amiRNA's ability to recognize its target mRNA to direct RNA silencing, then an increase in target expression (Fig. 2) and no change in the level of amiRNA accumulation (Fig. 3 ) would have been detected. However, Figure 3 clearly shows that this was not the mechanism responsible for the reduction in RNA silencing efficiencies of any of the mismatched amiRNA duplexes analyzed in this study.

The silencing efficiencies of three additional amiRNA duplexes were also assessed. AmiRNA duplexes SC-7-SC-9 were designed so that the preferentially selected duplex strand perfectly complemented the sequence of the targeted transgene (Fig. 1C). For strand choice duplexes SC-7 and SC-8, the G:U wobble pair at the $5^{\prime}$ terminal position of the antisense and sense strands of amiRNA duplexes SC-1 and SC-2 was replaced with a C-A mismatch. The introduction of this mismatched dsRNA base-pairing not only allowed for the preferentially selected strand to possess a $\mathrm{C}$ at its $5^{\prime}$ terminus and to exactly match its target sequence, but such a design also maintained the asymmetry of amiRNA duplexes SC-1 and SC-2. The introduction of a C-A mismatch at the $5^{\prime}$ end of the antisense strand of amiRNA duplex SC-7 triggered this duplex strand to function at the expense of the other, leading to efficient silencing of the GUS sense target transgene (Fig. 2G). Repositioning the C-A mismatch to the $5^{\prime}$ end of the sense strand in amiRNA duplex SC-8 dramatically enhanced silencing of the GUS antisense target transgene (Fig. $2 \mathrm{H}$ ), reversing the silencing efficiencies directed by SC-7. As shown for SC-6 (Fig. 2F), the symmetry expressed by amiRNA duplex SC-9 resulted in equivalent silencing of both versions of the target transgene (Fig. 2I), which correlated with relatively equal accumulation of both the sense and antisense strandspecific amiRNAs (Fig. 3). 


\section{The involvement of DRB1 and AGO1 in miRNA guide strand selection from miRNA duplexes}

The biased selection and processing of the amiRNA duplex strand with a lower thermodynamic stability at its $5^{\prime}$ end, regardless of the $5^{\prime}$ terminal nucleotide, to guide RNA silencing led us to investigate the roles specified by the miRNA biogenesis machinery proteins, DRB1 and AGO1, and to ask whether DRB1 functions more similarly to Loqs or R2D2. In Drosophila sRNA biogenesis, Loqs is required by Dcr-1 for efficient processing of certain classes of premiRNAs into mature miRNAs, but is dispensable for miRNA RISC loading (Saito et al. 2005; Liu et al. 2007), whereas Dcr-2 is able to process dsRNA templates in the absence of R2D2, but requires this dsRBP to form the RLC, which thermodynamically orientates siRNA duplexes onto Ago2 for passenger strand cleavage and active siRNAloaded RISC formation (Liu et al. 2003; Tomari et al. 2004). We included the Arabidopsis AGO1 mutant (ago1) in our analyses to determine if DRB1 was in fact operating in a similar manner to R2D2, orientating miRNA duplexes onto AGO1 for passenger strand cleavage. If in Arabidopsis DRB1 is indeed functioning in a similar fashion to the Drosophila dsRBP, R2D2, to direct loading of DCL1processed miRNA duplexes to RISC for passenger strand cleavage via the Slicer activity of AGO1, then we might expect to detect an increased accumulation of the miRNA* strand in both the drb1 and agol mutant backgrounds, compared with wild-type plants.

The levels of two well studied endogenous miRNAs, miR159 and miR167, and their respective miRNA* sequences were assessed (Fig. 4B) in wild-type (Col-0), $d r b 1$, and ago1 Arabidopsis plants. Both miRNAs accumulated to high levels in wild-type extracts, but their accumulation was reduced, although at detectable levels, in $d r b 1$ plants, as previously reported (Han et al. 2004; Vazquez et al. 2004). The miR159* and miR167* sequences were at undetectable levels in the Col-0 background; however, they accumulated to higher, readily detectable levels in the absence of DRB1. These results confirm the in vitro results of Dong et al. (2008), that DCL1 is capable in the absence of DRB1 of cleaving miRNA/miRNA ${ }^{\star}$ duplexes from their precursor transcript, and also show that the normal bias of miRNA guide strand accumulation is lost in the drbl mutant background. The simplest explanation of this result is that DRB1, like R2D2 in Drosophila, is responsible for strand bias by directionally loading miRNA/miRNA* duplexes onto AGO1-catalyzed RISC for passenger strand degradation (Fig. 7A, see below), and that in the absence of DRB1, duplexes are loaded without polarity selection.

A similar loss of miRNA/miRNA* polarity was observed in the ago1 mutant background. Figure $4 \mathrm{~B}$ shows that the accumulation of miRNA guide and passenger strands was decreased and enhanced, respectively, for both miR159 and miR167 in ago1 plants compared with wild type. This suggests that either the endonucleolytic activity of AGO1 is required for miRNA/miRNA* strand biasing, or that in the absence of AGO1, miRNA duplexes are loaded to another AGO effector complex that is not influenced by DRB1's directed strand selection. Vaucheret et al. (2004) have previously shown that expression of the Agol transcript is regulated by miR168, and we therefore may expect to see a unique accumulation profile for this endogenous miRNA. Northern blot analysis of the accumulation of this miRNA indeed revealed an interesting guide and passenger strand accumulation profile, as shown in Figure 4B. The accumulation of miR168 was largely unaffected in $d r b 1$, showing an equivalently high level of accumulation as that detected in wild-type plants. In addition, a reduction in the level of miR168* was detected by Northern blotting. Taken together, the miRNA/miRNA* duplex strand accumulation profile of miR168 in the $d r b 1$ mutant background directly contrasts those of the other two endogenous miRNAs analyzed to suggest that this miRNA is processed in a DRB1-independent manner. The accumulation of both miR168 duplex strands was reduced in the ago1 mutant background compared with their level in wild-type plants (Fig. 4B). In addition, the expression of Ago1 mRNA, the target of miR168-directed silencing, was almost below the level of detection by qRT-PCR in ago 1 (Fig. 4C). Reduced accumulation of both duplex strands for miR168 was not unexpected in this ago1 T-DNA insertion mutant knockdown line, as Vaucheret et al. (2006) have previously proposed a post-transcriptional auto-regulatory loop between miR168 and AGO1. That is, when Ago1 mRNA expression is reduced, leading to a decrease in AGO1 protein, miR168 is destabilized, resulting in its decreased accumulation and thus ensuring AGO1 homeostasis.

\section{Does an additional AGO protein express Slicer activity in the absence of AGO1?}

Quantitative RT-PCR with primers specific for the Arabidopsis Myb33, Arf8, and Ago1 transcripts was used to assess target expression of the miR159, miR167, and miR168 miRNAs, respectively. In the $d r b 1$ mutant background, the expression of miRNA target transcripts Myb33, Arf8, and Agol was up-regulated $\sim 2.9-, 1.7-$, and 1.3-fold, respectively, compared with their expression in wild-type plants (Fig. 4C). Surprisingly, the up-regulation of target transcript expression was not as dramatic in agol as in drb1 plants, with the targets for miR159 and miR167namely, Myb33 and Arf8-being up-regulated by $\sim 1.4$ and 1.2-fold, respectively. Consistent with this, comparison of miRNA accumulation to target transcript expression strongly suggested a correlation between miRNA accumulation and the level of target transcript expression among $d r b 1$, ago1, and wild-type plants. These results suggest that miR159 and miR167 can guide transcript silencing in the 
A
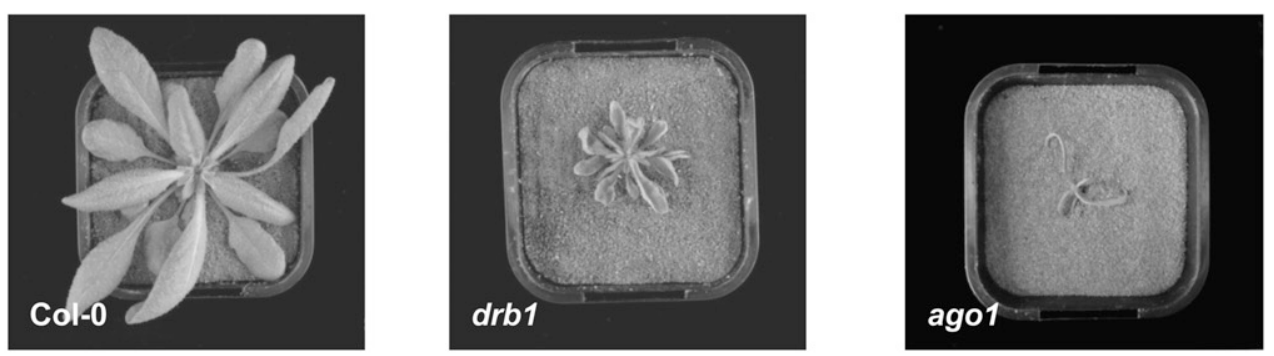

B


miR159*

U6

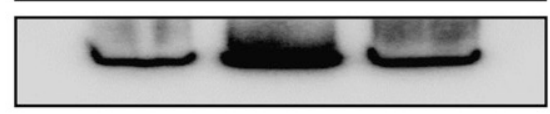

U6

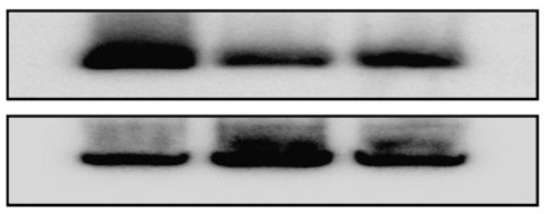

\section{miR167}

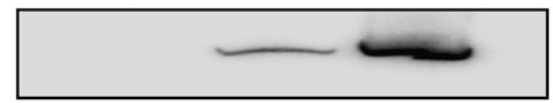

miR167*

U6

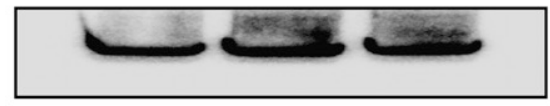

U6

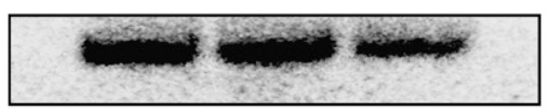

miR168

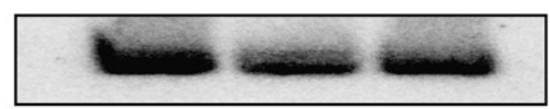

miR168*

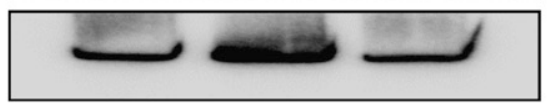

U6



U6

C
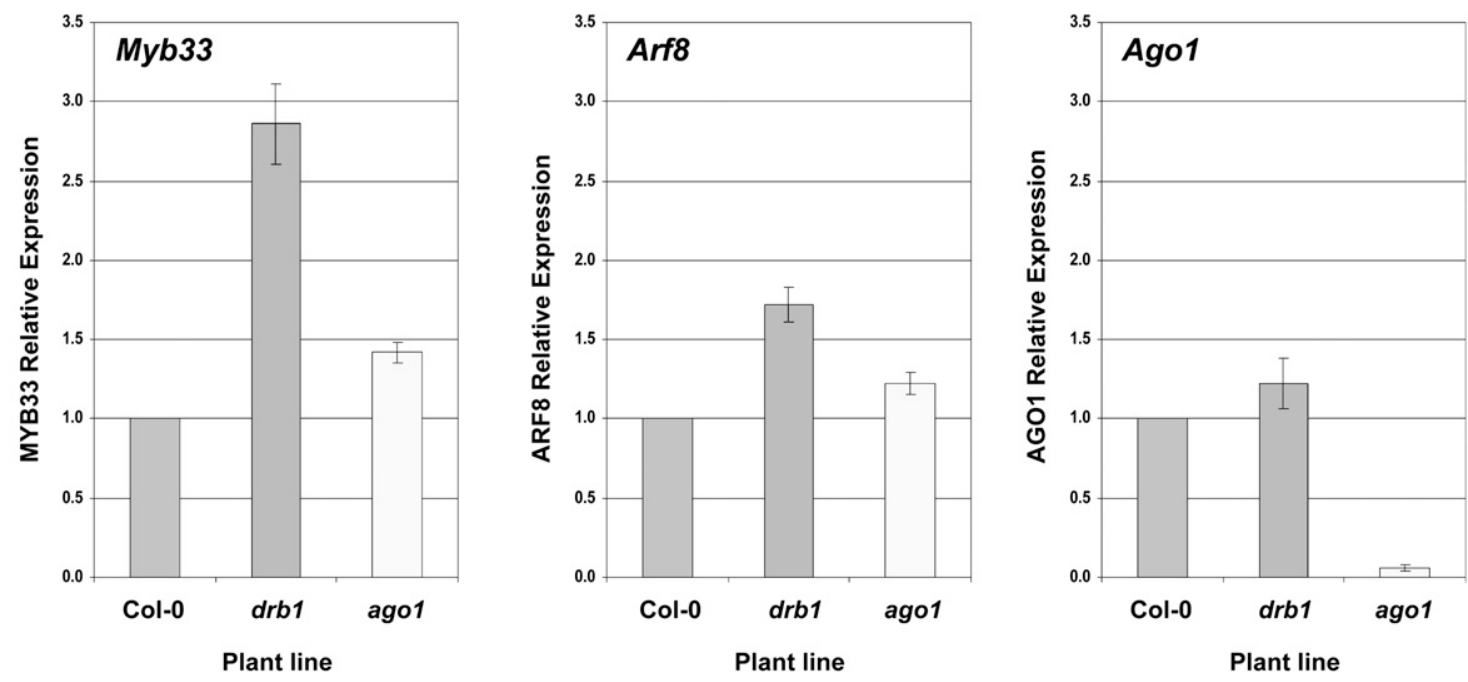

FIGURE 4. Phenotypes, sRNA accumulation, and target transcript expression in wild-type Arabidopsis plants and in drb1 and ago1 T-DNA insertional mutant knockout lines. (A) Phenotypes expressed by 4 -wk-old wild-type Arabidopsis plants (Col-0) and drb1 and ago1 T-DNA insertional mutant knockout lines. (B) Small RNA accumulation of miR159, miR167, and miR168 duplex strands (miRNA and miRNA*) in wildtype, $d r b 1$, and ago1 plant lines. (C) Transcript expression in wild-type, drb1, and ago1 plant lines for endogenous mRNAs, Myb33, Arf8, and Ago1, the target transcripts for miRNAs miR159, miR167, and miR168, respectively. Error bars represent the standard error of the mean (SEM) among three biological replicates. 
absence of AGO1, presumably by interaction with one of the other nine Arabidopsis AGO proteins.

\section{Endogenous miRNAs processed from Class II duplexes require DRB1, but miR168 does not}

Of the three endogenous miRNAs initially studied, Northern blot analysis showed that miRNAs processed from Class I duplexes-namely, miR159 and miR167-are preferentially selected over the duplex passenger strand, and that this preferential selection was lost in the $d r b 1$ mutant. However, the third miRNA analyzed is processed from a Class II duplex, where $5^{\prime}$ thermodynamic stability has little or no influence on strand selection. Northern blot analyses revealed that miR168 accumulated to near wildtype levels in the $d r b 1$ mutant background (Fig. 4B), suggesting that this endogenous miRNA, processed from a Class II miRNA duplex, does not require the coordinated action of DRB1 for efficient processing of its pri- and premiRNA transcripts by DCL1, or for directing the preferential selection of one duplex strand over the other. Therefore, we were interested in determining what influence DRB1 has on the biogenesis of other miRNAs processed from Class II duplexes. The accumulation of three additional miRNA families, including miR163, miR169, and miR408, processed from Class II duplexes (according to sRNA sequencing data that showed a "less" strongly biased number of reads for both strands) was also analyzed by Northern blotting. The accumulation of these three nonselected miRNA families was indeed reduced in drb1 (Fig. 5B), as was shown for four additional miRNA families analyzed by Northern blotting-namely, miR160, miR161, miR164, and miR172 (Fig. 5C) -all of which are processed from Class I miRNA duplexes that are influenced by the thermodynamic properties of duplex strands. Furthermore, and as shown for Class I miRNAs, miR159 and miR167 (Fig. 4B), the miRNA* sequence accumulated to higher, and in the majority of cases, detectable, levels in drb1 plants for five (miR160, miR161, miR164, miR172, and miR408) out of the seven additional miRNAs analyzed, including the Class II miRNA, miR408 (Fig. 5B). Figure 5B also shows that the miRNA* sequence processed from Class II miRNA duplexes, miR163 and miR169, remained below the level of detection by Northern blotting in the drb1 mutant background. This was not unexpected, as according to our sRNA sequencing data (Supplemental Data 1), miR163* and miR169* were detected three and 17 times, respectively, and, therefore, if their accumulation is enhanced in the absence of DRB1, then such a change may still fall below the level of detection by Northern blotting. Overall, these results strongly suggest that the majority of plant miRNAs require the coordinated action of DRB1 with DCL1, not only for efficient processing from their respective pri- and pre-miRNA transcripts (Han et al. 2004; Vazquez et al. 2004; Dong et al. 2008), but also to ensure the correct miRNA duplex strand, the miRNA guide strand, is selected for RISC incorporation. This result also suggests that the steps involved in the biogenesis of miR168 differ from those of the established plant miRNA biogenesis pathway.

\section{The preferential selection of miRNA guide strands is enhanced in the absence of DRBs 2, 3, 4, and 5}

The biased accumulation of miRNA duplex strands was also assessed in our dsRBP combinational insertion mutant knockout line, drb2345 (Curtin et al. 2008). In this line, only one, DRB1, of the five known Arabidopsis dsRBPs is functional. We were interested to see what effect this would have on DRBl's guided selection of the miRNA strand from Class I and Class II miRNA duplexes. In the absence of DRB2, 3, 4, and 5, the miRNA guide strand accumulated to higher than wild-type levels for all of the miRNAs analyzed (except miR168), regardless of the class of duplex from which the miRNA is processed (Fig. 5). In addition, and in direct correlation with the increased accumulation of miRNA guide strands, qRT-PCR analysis of miRNAregulated transcript expression (Fig. 5C) showed that the mRNAs for two miRNA targets-namely, Arf17 (miR160) and Cuc2 (miR164) - were down-regulated in the quadruple mutant. These results suggest that one or more of the four other dsRBPs competes with DRB1 for interaction with DCL1, or for the miRNA precursor molecules, and that, in their absence, DRB1 has exclusive access to its preferred partner (DCL1) and/or substrate.

\section{Strand selection of sRNAs produced by DCL4/DRB4}

In contrast to the majority of plant miRNAs generated by the DCL1/DRB1-directed biogenesis pathway, miR822 and miR839 rely on DCL4 for their accumulation (Rajagopalan et al. 2006). Furthermore, DCL4 exclusively interacts with DRB4, to efficiently process both viral and trans-acting siRNAs from their dsRNA substrates (Hiraguri et al. 2005; Adenot et al. 2006; Nakazawa et al. 2007; Curtin et al. 2008). Therefore, we studied the accumulation of these two miRNAs and their respective miRNA ${ }^{*}$ sequences in $d r b 1$ and $d r b 4$ mutant plant lines (Fig. 6A). If DRB4 is required by DCL4 for miRNA processing, then we would expect to detect a decrease in the accumulation of miR822 and miR839 in the drb4 mutant. Furthermore, if DRB4 functions in a similar manner to DRB1, to guide the preferential selection of one miRNA duplex strand over the other, then we would expect to see an increase in the accumulation of the miRNA* strands in $d r b 4$ plants. The accumulation of these two DCL4-dependent miRNAs was indeed reduced in $d r b 4$ (Fig. 6B). However, the accumulation of miR822* and miR839* appeared to be unchanged in the same mutant background, remaining below the level of detection in all three plant lines analyzed. The reduced 
A


B



C
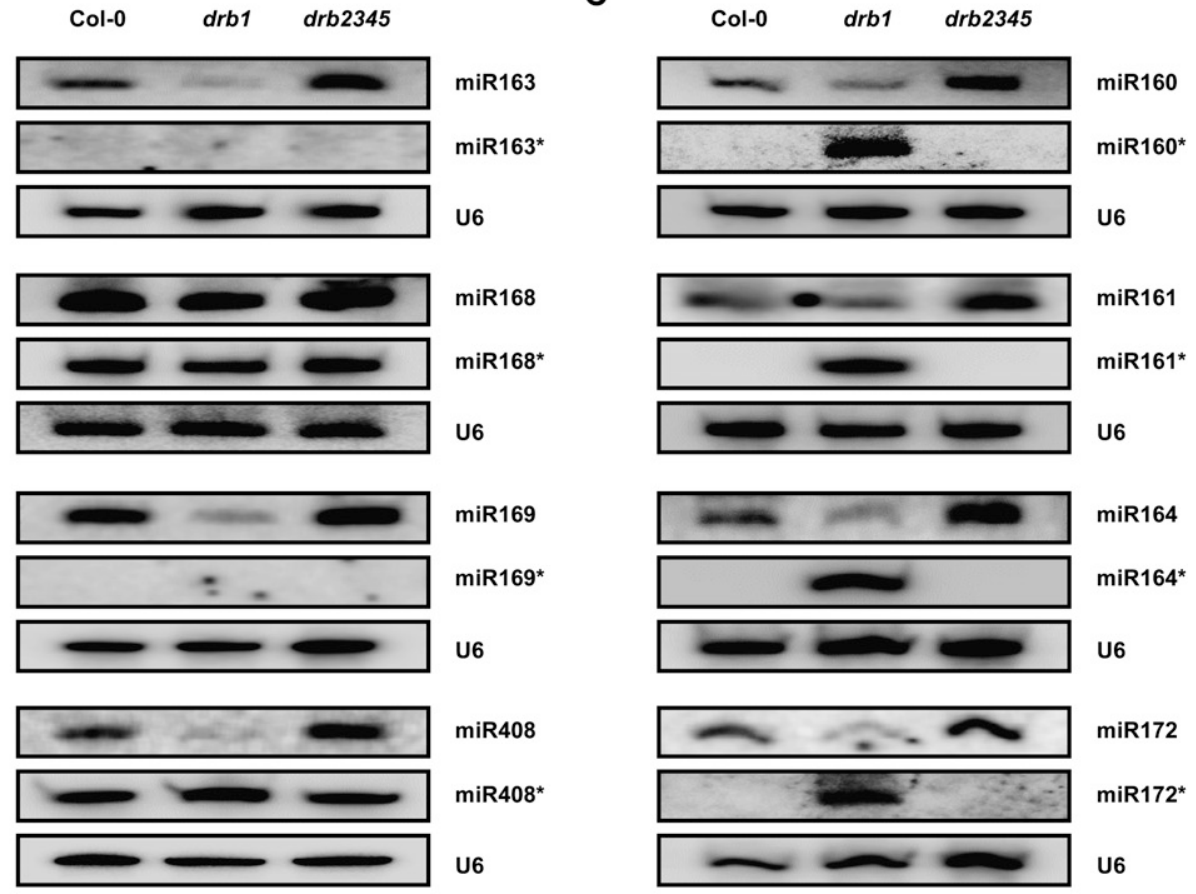

D
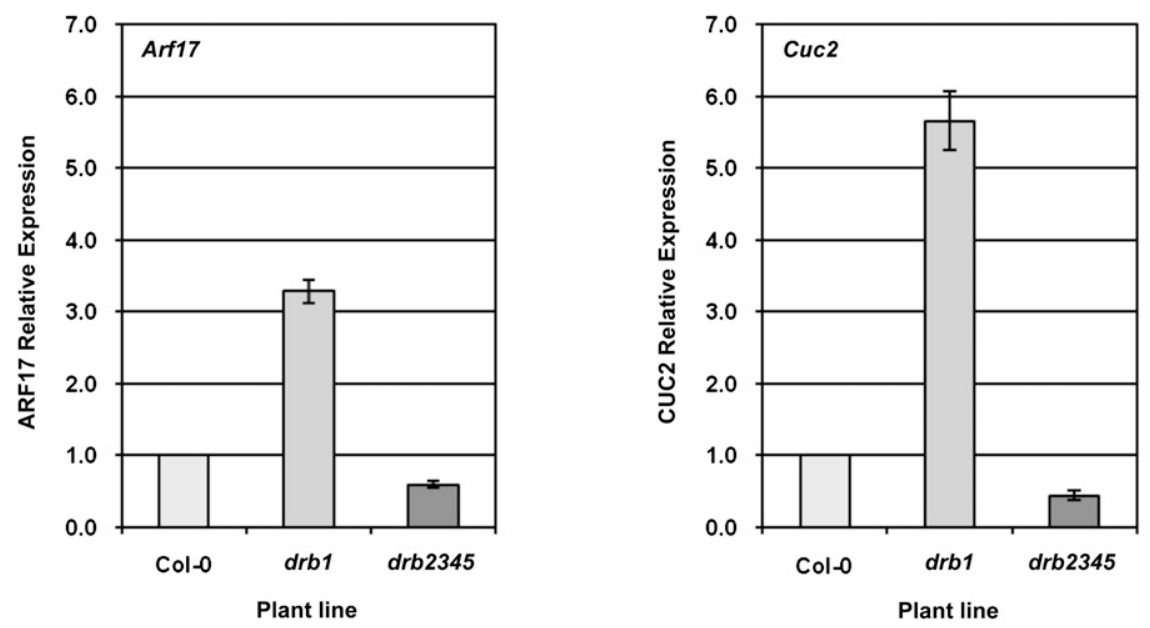

FIGURE 5. Phenotypes, sRNA accumulation, and target transcript expression in wild-type Arabidopsis plants and $d r b 1$ and $d r b 2345$ mutant lines. (A) Phenotypes expressed by 4-wk-old $d r b 1$ and $d r b 2345$ mutant lines compared with wild-type Arabidopsis plants (Col-0). (B) MicroRNA duplex strand accumulation (miRNA and miRNA*) in wild-type plants (Col-0) and in $d r b 1$ and $d r b 2345$ mutant lines for miR163, miR168, miR169, and miR408, Arabidopsis miRNAs processed from Class II miRNA duplexes. (C) MicroRNA duplex strand accumulation (miRNA and miRNA ${ }^{\star}$ ) in T-DNA insertional mutant knockout lines $d r b 1$ and $d r b 2345$, compared with wild-type (Col-0), for miR160, miR161, miR164, and miR172, endogenous miRNAs processed from Class I miRNA duplexes. (D) Target transcript expression of miRNA-regulated mRNAs, Arf17 (miR160; processed from a Class II miRNA duplex) and Cuc2 (miR164; processed from a Class I miRNA duplex), in wild-type (Col-0), drb1, and $d r b 2345$ plants. Error bars represent the standard error of the mean (SEM) among three biological replicates. 
A
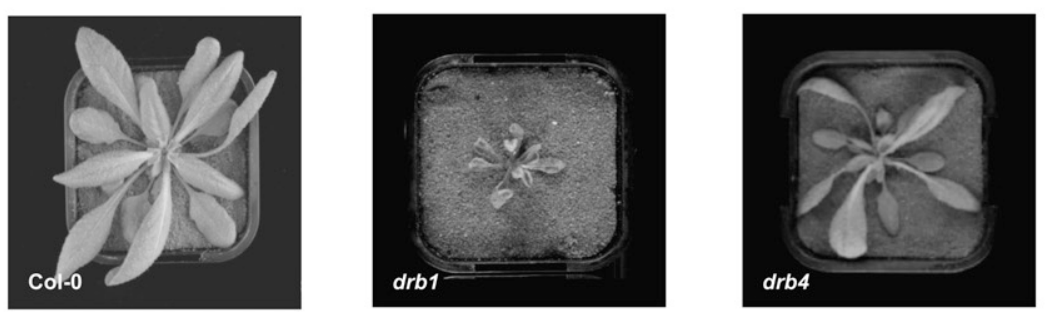

B
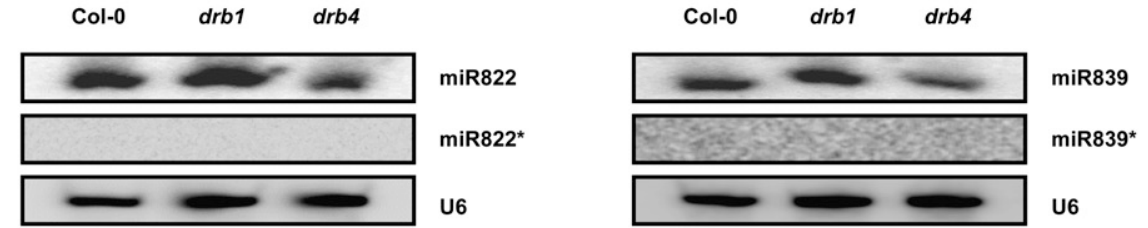

C



FIGURE 6. DCL4/DRB4-dependent sRNA accumulation in wild-type Arabidopsis plants and in drb1 and drb4 T-DNA insertional mutant knockout lines. (A) Phenotypes expressed by 4-wk-old wild-type (Col-0), drb1, and drb4 plants. (B) MicroRNA duplex strand accumulation (miRNA and miRNA ${ }^{*}$ of DCL4/DRB4-dependent miRNAs, miR822 and miR839, in wild-type Arabidopsis plants, and in drb1 and drb4 T-DNA insertional mutant knockout lines. $(C)$ The abundance ratios (as percentages) of siRNA duplex strands derived from the introduced GFP hpRNA, and endogenous Arabidopsis $\mathrm{miR} / \mathrm{miR}^{\star}$ duplex strands. All possible siRNA duplexes that could be generated from the GFP hpRNA were categorized by their $5^{\prime}$ terminal nucleotide ( $5^{\prime}$ terminal nucleotides of duplex strands designated $\mathrm{X}$ and $\mathrm{Y}$ ). For siRNAs, each graphed point is calculated using the formula $\mathrm{X}$ strand $\mathrm{X}+\mathrm{Y}$ strand $(\times 100)$. For miRNAs, the same formula is used where $\mathrm{X}=$ miRNA strand and $\mathrm{Y}=$ miRNA strand. (Filled diamonds) siRNAs or miRNAs with the abundance predicted by the miRNA termini thermostability rules, (open diamonds) siRNAs or miRNAs present at abundances contrary to predictions based on the miRNA termini thermostability rules. (M) Mismatched bases, (W) weak $\mathrm{G}-\mathrm{U}$ base pairing.

accumulation of the miRNA guide strand for these two DCL4-dependent miRNAs suggests that DRB4 is required by DCL4 for their efficient biogenesis, but, unlike DRB1,
DRB4 is not responsible for the preferential selection of one duplex strand over the other, and that the biased accumulation of the guide strand over the corresponding passenger 
strand from these two DCL4-processed miRNA duplexes is regulated via a different mechanism than DCL1/DRB1dependent miRNAs.

The asymmetry of synthetic or endogenous siRNA (namely, tasiRNAs) duplexes was originally proposed to influence strand selection (Khvorova et al. 2003; Vazquez et al. 2004). However, Rajagopalan et al. (2006) subsequently demonstrated that there was no correlation between the preferential selection of one duplex strand and the thermodynamic properties expressed by the selected strand from siRNA duplexes. Therefore, to assess whether strand selection of siRNAs mirrored that of DCL4/ DRB4-generated miRNAs (Fig. 6B), or if siRNA strand selection followed the same thermostability rules as those governing the DRB1-directed selection of artificial or endogenous miRNAs, a transgene encoding hpRNA (Fig. 1D) derived from the $5^{\prime}$ half of the GFP gene was transformed into Arabidopsis, and the sRNAs produced in these plants were analyzed by deep sequencing. The abundance of the siRNAs derived from the hpRNA and those of 58 endogenous miRNAs and their counterpart miRNA ${ }^{\star}$ s were evaluated by the number of times each molecule was sequenced. The relationship, expressed as a percentage, between the number of reads for each hpRNA-derived topstrand siRNA and its bottom-strand counterpart (assuming DCL4 dices to generate 21-nt duplexes with 2-nt 3' overhangs) and the percentage ratio of reads for each miRNA and its counterpart miRNA ${ }^{*}$ strand are shown in Figure $6 \mathrm{C}$. The results demonstrate that while there is bias toward retention of the strand with weaker $5^{\prime}$ terminal thermostability for most DCL1/DRB1 generated miRNAs, this is not generally the case for siRNAs produced from hpRNA by DCL4/DRB4. For example, in Figure 6C, the top line of points represents 15 different siRNA duplexes, each with one strand possessing a $5^{\prime}$ terminal adenine residue and the other strand starting with a $5^{\prime}$ uracil. Such duplexes have symmetrical $5^{\prime}$ terminal thermostability, and, according to strand selection rules from miRNA duplexes processed by DCL1/DRB1, we would expect to see equal accumulation of both siRNA strands. However, while a few of the siRNA/ siRNA* pairs show relatively unbiased strand selection consistent with these rules, most siRNA/siRNA ${ }^{\star}$ pairs show pronounced bias for the retention of one siRNA duplex strand. Similarly, duplexes expected from the DCL1/DRB1 miRNA rules to show pronounced bias toward one strand (i.e., with one strand commencing with a uracil and the other with a guanine) have many instances with strong bias to the counterpredicted strand.

\section{DISCUSSION}

Bioinformatic analysis of individual miRNAs present in our sRNA-specific sequencing population suggested that the majority of Arabidopsis miRNAs are preferentially selected over their miRNA* strands due to the asymmetric thermo- dynamic stability of the miRNA/miRNA* duplex termini. Our experiments, using amiRNAs, directly tested this hypothesis and demonstrated that miRNA strand selection in plants is directed in this way. The strand of the amiRNA/ amiRNA* duplex with a weaker $5^{\prime}$ dsRNA base-pairing was repeatedly shown to be preferentially selected for retention by RISC and used to direct RNA silencing. In addition to testing termini with differing thermodynamic properties, we also analyzed amiRNA/amiRNA* duplexes designed to possess uracil, adenine, or cytosine residues at their $5^{\prime}$ termini, as AGO proteins 1, 2, and 5 have been shown to have preferences for sRNA molecules with $\mathrm{U}, \mathrm{A}$, and $\mathrm{C}$ at their $5^{\prime}$ ends, respectively ( $\mathrm{Mi}$ et al. 2008; Takeda et al. 2008). Strand choice duplexes, SC-1-SC-3, were designed to have uracil at the $5^{\prime}$ end of the selected duplex strand, whereas duplexes SC-4-SC-6 possessed adenine at this position, and duplexes SC-7-SC-9 had cytosines at their $5^{\prime}$ termini. Changing the $5^{\prime}$ terminal nucleotide of GUS strand choice duplexes, but maintaining a duplex's symmetry of thermodynamic terminal stability, did not affect the silencing efficiencies or polarities of any of the nine amiRNAs analyzed in this study. Furthermore, amiRNA duplex SC-3, which has a uracil residue at position 1 of both duplex strands to direct loading of the processed strand to AGO1, showed strong bias for retention of the strand with the mismatching $5^{\prime}$ end, resulting in high degrees of RNA silencing of the corresponding GUS target transgene. Overall, the results of our amiRNA strand selection experiments directly show that the thermodynamic properties of a miRNA/miRNA* duplex are the crucial determinant for RNA silencing efficiency, not the nucleotide at the $5^{\prime}$ terminus of the selected strand.

The coordinated action of DRB1 (HYL1) and DCL1 is known to be required for the efficient processing of primiRNA and pre-miRNA transcripts for normal miRNA accumulation in Arabidopsis (Han et al. 2004; Vazquez et al. 2004; Kurihara et al. 2006; Dong et al. 2008). Here, we show that DRB1 plays an additional role in the Arabidopsis miRNA biogenesis pathway. Northern blot analyses of guide and passenger strand accumulation in $d r b 1$ plants, initially of two well characterized endogenous miRNAs, revealed that miRNA or guide strand accumulation is reduced in this mutant background, as previously reported (Han et al. 2004; Vazquez et al. 2004). However, we also demonstrate that the miRNA* sequences for these two, and for an additional five endogenous miRNAs, are up-regulated in the same mutant background compared with their respective accumulation levels in wild-type plants. Dong and colleagues (2008) showed from in vitro experiments that in the absence of DRB1, DCL1 processing of a synthetic pri-miR167b transcript is inaccurate, with the majority of sRNAs processed from this dsRNA precursor mapping to the molecule's $3^{\prime}$ end. The accumulation of these inaccurately processed DCL1 cleavage products would not have influenced our Northern blotting assessments of miRNA 
and miRNA $^{*}$ accumulation in the drbl mutant background, as the sRNA-specific (miRNA or miRNA*) 21-nt radiolabeled DNA oligonucleotide probes used in this study would not detect such sRNAs. Instead, we favor the hypothesis that the residual amount of accurately processed miRNA/miRNA* duplex that accumulates in $d r b 1$ mutants is no longer being directionally loaded onto AGO1 for passenger strand degradation, thereby resulting in an increased and equivalent expression of the miRNA* strand. We therefore propose that in the Arabidopsis miRNA biogenesis pathway, DRB1 acts in a similar manner to R2D2 in the Drosophila siRNA biogenesis pathway (Tomari et al. 2004; Matranga et al. 2005; Rand et al. 2005), by binding to or interacting with the more thermodynamically stable end of the miRNA duplex to orientate the duplex for AGO-mediated passenger strand cleavage of the miRNA* moiety.

As expected, the mRNA levels of Myb33 and Arf 8 in both the $d r b 1$ and agol mutant backgrounds were higher than in wild-type plants and correlated with decreased levels of their regulating miRNAs. However, the release of this miRNA control was less pronounced in the agol background. This may be due to drb1 being a null mutation, while ago1 is a hypomorphic mutant with reduced but measurable levels of AGO1 expression. However, it is also possible that a protein encoded by one or more of the nine other AGO genes in Arabidopsis may also be capable of AGO1-like miRNA-directed Slicer activity, and that this only becomes obvious when AGO1 levels are reduced. Such a hierarchy of function has been observed for the DCL gene family (Gasciolli et al. 2005; Xie et al. 2005; Blevins et al. 2006; Deleris et al. 2006; Fusaro et al. 2006). It is interesting that some of the amiRNAs used in this study, with $5^{\prime}$ terminal adenine and cytosine residues, are highly effective. This suggests either that AGO1 can also be efficiently loaded with amiRNA guide strands that have $5^{\prime}$ terminal bases other than uracil, or that other Argonautes such as AGO2 and AGO5 are able to use mature miRNAs as guides to direct RNA silencing.

Compared with wild type, the levels of almost all miRNAs are reduced, and their target mRNA levels are elevated, in both agol and dcl1 mutant plants (Park et al. 2002; Vaucheret et al. 2004). However, the accumulation of miR168 is relatively unaffected in three hypomorphic $d c l 1$ alleles (Vaucheret et al. 2006), and we also show that the expression of both the guide (Vazquez et al. 2004; Curtin et al. 2008) and passenger strands (present study) of miR168 are relatively unaffected in the $d r b 1$ mutant background, accumulating to approximately wild-type levels. We also found that, unlike most miRNAs, the strand selection of miR168 from its miRNA/miRNA ${ }^{*}$ duplex is not influenced by differential thermostability of each strand's $5^{\prime}$ termini. However, one other miRNA, miR408, which also appears to exhibit independence from differential thermostability for strand selection, does require DRB1 for its production and/or maturation. Taken together, these results suggest that miR168 is a uniquely processed miRNA. Perhaps this situation has evolved to ensure that miR168, and hence, AGO1, is maintained at a normal level, even in circumstances that detrimentally affect other miRNA and sRNA regulated pathway components, thereby maintaining some degree of homeostasis.

In plants lacking all of the DRB family members except DRB1, miRNA guide strands accumulated to higher than wild-type levels. However, in the same mutant background the accumulation of the corresponding duplex strand, the miRNA ${ }^{*}$ strand, remained unchanged. This enhanced selection of the miRNA guide strand was repeatedly demonstrated in the $d r b 2345$ quadruple mutant, regardless of the duplex class from which the miRNA is processed. A possible explanation is that DRB1, in wild-type plants, is in competition with one or more of the other four DRBs for interactions with DCL1 and/or miRNA precursor molecules, and that because none of these four dsRBPs are able to assist DCL1 in processing miRNA precursor molecules or in directing strand selection, the competition has a repressive effect on miRNA production. Kurihara and colleagues (2006) demonstrated that cleavage accuracy of pri-, pre-, and mature miRNA molecules varied for three individual miRNAs analyzed (miR163, miR164b, and miR166a) in $d c l 1$ and $d r b 1$ mutants, suggesting that misplaced cleavage does not solely depend on the dysfunction of DCL1 or DRB1, but is also influenced by the substrate's dsRNA structure itself, or on the interaction of other dsRBPs with DCL1. Hiraguri et al. (2005) showed that the DRB1 protein interacts with DCL1 through one of its two adjacent dsRNA binding domains (dsRBDs), located in the N-terminal half of the protein. All five DRB proteins contain adjacent dsRBDs in their $\mathrm{N}$ termini (Curtin et al. 2008), giving each of them the potential to interact with DCL1 and/or its substrates. DRB2 and DRB5 bind to DCL1 in vitro, but do not show a preference for DCL1 over DCLs 2, 3, or 4 (Hiraguri et al. 2005). However, this interaction with DCL1 identifies them as strong candidates for competitors to DRB1 for partnership with DCL1 in the miRNA biogenesis pathway and suggests possible roles for these proteins as regulators of miRNA production.

Overall, our results clearly demonstrate that DRB1 is required to direct strand selection from DCL1-processed miRNA duplexes. Furthermore, AGO1 appears to be responsible for degradation or destabilization of the nonselected duplex strand. In Arabidopsis, the Slicer activity associated with miRNA-loaded RISC specifically co-elutes with small protein complexes (Baumberger and Baulcombe 2005), suggesting that the minimal miRNA-loaded RISC may contain only AGO1 and its associated sRNA. In addition, DRB1 and DCL1 have been shown to co-localize to nuclear dicing bodies or D-bodies (Song et al. 2007), and that additional miRNA-processing machinery, including 
HEN1, SE, and AGO1, also show some localization to the nucleus and to D-bodies (Fang and Spector 2007). Integrating this information with our results allows us to propose a model (Fig. 7) describing the first steps of miRNA production and processing in the nucleus and subsequent release into the cytoplasm of a miRNA-loaded AGO1. Our model suggests that following the processing of miRNA primary and precursor transcripts and the methylation of the duplex strand's 2-nt 3' overhangs by HEN1, DRB1 on its own or together with DCL1, forming a heterodimer similar to the RLC of the Drosophila siRNA biogenesis pathway, is responsible for orientating the miRNA duplex for passenger strand degradation via DRB1's interaction with the more thermodynamically stable end of the miRNA duplex. This small complex, either in D-bodies or in the nucleus itself, directionally loads the miRNA duplex onto AGO1, where the passenger strand is cleaved and released to produce a mature miRNA-loaded RISC. The act of miRNA duplex loading or passenger-strand cleavage may serve as a trigger for the active transport of miRNA-loaded AGO1 through nuclear pores into the cytoplasm where, directed by its guide strand, AGO1 cleaves cognate mRNAs.

Curiously, miRNAs processed from endogenous precursor transcripts and siRNAs generated from an introduced hpRNA by DCL4/DRB4 do not show a uniform bias toward retaining the duplex strand with the weaker $5^{\prime}$ terminal thermostability, as shown for the majority of DCL1/DRB1-generated miRNA/miRNA* duplexes detected in the same sRNA sequencing set. This suggests that the production and selection of sRNAs in the DCL1/DRB1 and DCL4/DRB4 pathways operate under different rules. Our results also demonstrate that the mechanisms governing miRNA and siRNA biogenesis in Arabidopsis directly contrast with sRNA biogenesis in Drosophila. In Drosophila, the RLC is a heterodimer composed of Dcr-2 and R2D2 and is responsible for orientating siRNA duplexes onto Ago 2 for passenger strand degradation; this preferential selection of the siRNA guide strand is directed by R2D2 binding to the more thermodynamically stable end of the siRNA duplex (Liu et al. 2003; Tomari et al. 2004). Surprisingly, in Arabidopsis, DRB1 and not DRB4 appears to function in a similar manner, influencing the retention of the less thermodynamically stable miRNA duplex strand by RISC to guide RNA silencing. Conversely, the Drosophila miRNA-associated dsRBP Loqs is required by Dcr-1 to efficiently process pre-miRNA transcripts into miRNA duplexes (Förstemann et al. 2005; Jiang et al. 2005; Saito et al. 2005), but unlike the requirement of R2D2 by Dcr-2 to form the RLC during siRNA biogenesis, Dcr-1 does not require Loqs for miRNA-loaded RISC assembly (Liu et al. 2007). In Arabidopsis the Dcr-1/Loqs partnership is mirrored by DCL4/DRB4, in that DRB4 is required by DCL4 for the efficient production of sRNAs from dsRNA substrates, but DRB4 does not have the same influence on strand selection and RISC incorporation of DCL4-generated sRNAs.

Have the evolutionary requirements of regulating gene expression resulted in the mechanistic interchanging of these two parallel RNA silencing pathways between plants and animals? In Arabidopsis, the production of siRNAs by the combined action of DCL4 and DRB4 from hpRNAs and replicating viral RNAs closely mirror each other (Fusaro et al. 2006; Curtin et al. 2008). It is therefore possible that the rules governing the production of such sRNAs by DCL4/DRB4 reflect the evolutionary desirability of having siRNAs targeting both the plus and minus strands of a replicating virus. Conversely, miRNA biogenesis may have retained strand selection to ensure that each required silencing signal is produced at a specific point during plant development, to in turn ensure that normal gene expression is maintained. Alternatively, differences in sRNA strand selection may exist between these parallel RNA silencing pathways of Arabidopsis, and in other organisms, to minimize the competition between anti-viral siRNA and miRNAs, and between these individual sRNA classes

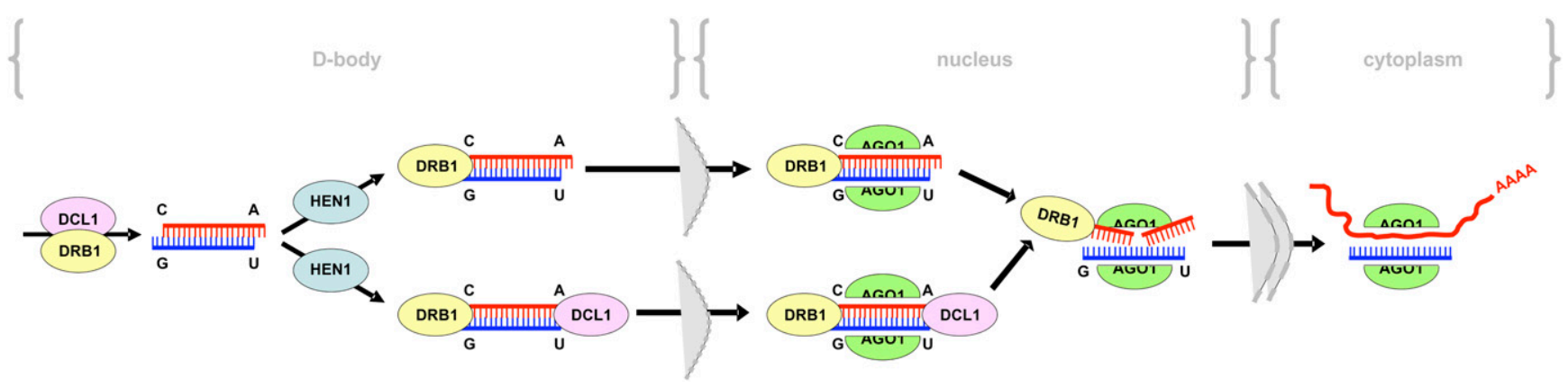

FIGURE 7. DRB1-directed selection of miRNA guide strands. The upper pathway shows a schematic working model for the Arabidopsis DRB1 protein functioning in a similar fashion to the Drosophila siRNA-specific dsRBP, R2D2, directing the loading of miRNA duplexes to AGO1 for passenger strand degradation through its preferential binding to the more thermodynamically stable end of miRNA duplexes. The lower pathway shows the alternate working model for the combined action of DRB1 with DCL1, forming a heterodimer, similar to the RLC of the Drosophila siRNA biogenesis pathway, directionally loading the miRNA duplex to AGO1 for passenger strand degradation. 
themselves, for loading into specific AGO effector complexes; however, the reasons for such mechanistic differences remain to be determined.

\section{MATERIALS AND METHODS}

\section{Vector construction}

Construction of the artificial miRNA (amiRNA) plant expression vector

The DNA sequence encoding the primary transcript (pri-miRNA) of the Arabidopsis MIR159b gene was amplified from genomic DNA using primers pMIR159-F and pMIR159-R (Supplemental Data 2) and cloned into the pGEM-T Easy vector (Promega) to produce pAth-miR159b. This vector was mutated using the Erasea-base system to generate SapI restriction sites flanking the precursor-miRNA (pre-miRNA) sequence of MIR159b. Using the SapI restriction sites, the pre-miRNA sequence was replaced with a similarly digested PCR fragment of the LacZ gene to produce pAth-miR159b/LacZ and allow for blue/white selection of bacterial transformants. Following EcoRI and HindIII digestion, the pre-miRNA/LacZ restriction fragment was cloned into the similarly digested pART7 shuttle vector (Gleave 1992) to flank this fragment with the $35 \mathrm{~S}$ promoter (35S-P) and OCS terminator (OCS-T) to produce pART7:Ath-miR159b/LacZ. The pART7:AthmiR159b/LacZ shuttle vector was digested with NotI to release the 35S-P:Ath-miR159b/LacZ:OCS-T fragment, which was cloned into the pGreen plant expression vector (Hellens et al. 2000), which had been mutated using the Erase-a-base system to remove the existing SapI restriction sites to create the amiRNA plant expression vector pBlueGreen. To generate the series of GUS strand choice plant expression vectors, the native miR159b and miR159b* sequences of pAth-miR159b were replaced with amiRNA and amiRNA* sequences using primers encoding fusions of these sequences with SapI restriction sites and the region complementary to the pre-miR159b flanking sequences by PCR (Supplemental Data 2). These PCR products were digested with SapI and ligated to the similarly digested plant expression vector pBlueGreen to replace the LacZ gene with the amiRNA premiRNA sequence. The resulting series of GUS strand choice plant expression vectors were used for transient expression in Nicotiana benthamiana leaves via Agrobacterium-mediated transformation.

Construction of plant expression vectors for the expression of the GUS sense and antisense targets and the GFP hairpin

The GUS sense target vector was constructed by PCR amplification of the $\beta$-glucuronidase (GUS) reporter gene from vector pEU334bn (Eamens et al. 2004) with primers pGUS-S-5 and pGUS-S-3 (Supplemental Data 2) flanked by EcoRI and HindIII restriction sites, respectively, and cloning into the pGEM-T Easy Vector to produce pGEM-T:GUS-S. pGEM-T:GUS-S was digested with EcoRI and HindIII to produce a GUS sense insertion fragment that was cloned into the similarly digested shuttle vector pART7 (Gleave 1992) to produce pART7:GUS-S. pART7:GUS-S was digested with NotI to release a 35S-P:GUS-S:OCS-T restriction fragment that was cloned into the similarly digested plant expression vector pART27 (Gleave 1992) to produce
pART27:GUS-S. The GUS antisense target vector pART27:GUSAS was constructed as outlined for pART27:GUS-S except primers pGUS-AS-5 and pGUS-AS-3 (Supplemental Data 2) were used to amplify and orientate the GUS PCR insertion fragment in the antisense direction. The steps involved in the construction of the GFP hairpin RNA plant expression vector (pUQ251) have been described previously (Brosnan et al. 2007).

\section{Plant transformation and growth conditions}

Transient expression of amiRNA vectors in $\mathrm{N}$. benthamiana

The amiRNA strand choice (SC-1-SC-9) and target vectors (GUS sense or antisense transgene) were transformed into Agrobacterium (strain GV3101) by electroporation. Resistant colonies were subcultured in Luria-Bertani (LB) media containing the appropriate selection and cultured for $48 \mathrm{~h}$ at $28^{\circ} \mathrm{C}$. Agrobacterium cultures were pelleted by centrifugation and resuspended in infil-

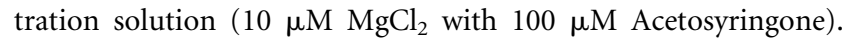
Mixed cultures of Agrobacterium containing each of the nine amiRNA strand choice vectors and either the GUS sense or antisense target transgene were inoculated into young developing leaves of $N$. benthamiana plants ( $\sim 3$ wk of age) that were grown under standard glasshouse conditions $\left(24^{\circ} \mathrm{C}\right.$ with $16 \mathrm{~h}$ of light and $8 \mathrm{~h}$ of dark) for $5 \mathrm{~d}$ post inoculation (dpi).

\section{Study of Arabidopsis wild-type and T-DNA insertional mutant knockout plant lines}

The Arabidopsis ago1 (SALK_116845; Alonso et al. 2003) T-DNA insertional mutant knockout line (ecotype Columbia-0) used in this study was identified using the TAIR SeqViewer T-DNA/ Transposon online search engine (http://www.arabidopsis.org/ servlets/sv) and seeds were ordered from the Nottingham Arabidopsis Stock Center (NASC). The identification and/or generation of Arabidopsis drb1 (hyl1-2; Vazquez et al. 2004), drb4 (drb4-1; Adenot et al. 2006), and $d r b 2345$ (Curtin et al. 2008) mutant lines, all in the Columbia-0 background, have been described previously. Arabidopsis seeds (wild-type and T-DNA insertional mutant knockout lines) were germinated and grown on Murashige and Skoog (MS) media containing the appropriate selection for $2 \mathrm{wk}$, and resistant seedlings were transferred to soil and grown under standard glasshouse conditions $\left(24^{\circ} \mathrm{C}\right.$ with $16 \mathrm{~h}$ of light and $8 \mathrm{~h}$ of dark) for an additional 2-wk period.

\section{RNA analyses}

qRT-PCR analysis of miRNA target transcript expression

Total RNA was extracted from $N$. benthamiana leaves 5 dpi with either the GUS sense or GUS antisense target transgene alone or in combination with each of the nine amiRNA strand choice vectors using TRIzol Reagent (Invitrogen). TRIzol Reagent was also used to extract total RNA from Arabidopsis wild-type plants and from T-DNA insertional mutant knockout lines. Total RNA $(50 \mu \mathrm{g})$ was digested with 10 units of RQ1 RNase-free DNase for $30 \mathrm{~min}$ at $37^{\circ} \mathrm{C}$ and then purified using an RNeasy Mini Kit (Qiagen). Purified RNA ( $5 \mu \mathrm{g}$ ) was used to synthesize cDNA with SuperScript III reverse transcriptase (Invitrogen). Diluted cDNA (1:20) was used as the template for qRT-PCR with $1 \times$ SYBR Green JumpStart Taq ReadyMix (Sigma) and primers specific for each transcript 
analyzed (Supplemental Data 2). The expression of each analyzed transcript was normalized to the Arabidopsis gene Cyclophilin (At2g29960). Error bars (Figs. 2, 4C, 5D) represent the standard error of the mean (SEM) among three biological replicates.

\section{Northern blot analysis of sRNA accumulation}

Northern blot analyses to assess miRNA accumulation in inoculated N. benthamiana leaves and from Arabidopsis plant lines were essentially performed as described previously (Jones et al. 2006). In brief, total RNA was isolated from plant tissues using TRIzol Reagent. Twenty micrograms of total RNA were separated on $17 \%$ denaturing ( $10 \mathrm{M}$ urea) polyacrylamide gels by electrophoresis and transferred to HyBond $-\mathrm{N}^{+}$membrane (Amersham) by electroblotting. DNA oligonucleotide probes specific for either artificial or endogenous miRNAs were $5^{\prime}$ end-labeled using T4 Polynucleotide Kinase and $\gamma-{ }^{32} \mathrm{P}$ ATP. All DNA oligonucleotide probes used in this study are listed in Supplemental Data 3.

\section{Small RNA sequencing}

Total RNA samples were extracted from Arabidopsis plants transformed with the GFP hpRNA construct pUQ251 (Brosnan et al. 2007) using TRIzol Reagent and were shipped to Illumina Pty for processing. The sequences of small RNAs (15-35 nt in length) were determined using Solexa technology as described previously (Zhu et al. 2008).

\section{SUPPLEMENTAL MATERIAL}

Supplemental material can be found at http://www.rnajournal.org.

\section{ACKNOWLEDGMENTS}

We thank Drs. Liz Dennis, Frank Gubler, and Adriana Fusaro for helpful suggestions throughout the course of this study and for critical reading of the manuscript, and Mr. Craig Jackson for his excellent technical assistance with the DRB4 small RNA Northern blots. This work was supported by the CSIRO Emerging Science Initiative.

Received March 12, 2009; accepted September 3, 2009.

\section{REFERENCES}

Adenot X, Elmayan T, Lauressergues D, Boutet S, Bouché $\mathrm{N}$, Gasciolli V, Vaucheret H. 2006. DRB4-dependent TAS3 transacting siRNAs control leaf morphology through AGO7. Curr Biol 16: 927-932.

Allen E, Xie Z, Gustafson AM, Carrington JC. 2005. MicroRNAdirected phasing during trans-acting siRNA biogenesis in plants. Cell 121: 207-221.

Alonso JM, Stepanova AN, Leisse TJ, Kim CJ, Chen H, Shinn P, Stevenson DK, Zimmerman J, Barajas P, Cheuk R, et al. 2003. Genome-wide insertional mutagenesis of Arabidopsis thaliana. Science 301: 653-657.

Bagga S, Bracht J, Hunter S, Massirer K, Holtz J, Eachus R, Pasquinelli AE. 2005. Regulation by let-7 and lin-4 miRNAs results in target mRNA degradation. Cell 122: 553-563.

Baumberger N, Baulcombe DC. 2005. Arabidopsis ARGONAUTE1 is an RNA Slicer that selectively recruits microRNAs and short interfering RNAs. Proc Natl Acad Sci 102: 11928-11933.
Blevins T, Rajeswaran R, Shivaprasad PV, Beknazariants D, SiAmmour A, Park HS, Vazquez F, Robertson D, Meins F Jr, Hohn T, et al. 2006. Four plant Dicers mediate viral small RNA biogenesis and DNA virus induced silencing. Nucleic Acids Res 34: 6233-6246.

Bouché N, Lauressergues D, Gasciolli V, Vaucheret H. 2006. An antagonistic function for Arabidopsis DCL2 in development and a new function for DCL4 in generating viral siRNAs. EMBO J 25: 3347-3356.

Boutet S, Vazquez F, Liu J, Béclin C, Fagard M, Gratias A, Morel JB, Crété P, Chen X, Vaucheret H. 2003. Arabidopsis HEN1: A genetic link between endogenous miRNA controlling development and siRNA controlling transgene silencing and virus resistance. Curr Biol 13: 843-848.

Brodersen P, Vionnet O. 2006. The diversity of RNA silencing pathways in plants. Trends Genet 22: 268-280.

Brosnan CA, Mitter N, Christie M, Smith NA, Waterhouse PM, Carroll BJ. 2007. Nuclear gene silencing directs reception of longdistance mRNA silencing in Arabidopsis. Proc Natl Acad Sci 104: 14741-14746.

Curtin SJ, Watson JM, Smith NA, Eamens AL, Blanchard CL, Waterhouse PM. 2008. The roles of plant dsRNA-binding proteins in RNA-like pathways. FEBS Lett 582: 2753-2760.

Deleris A, Gallego-Bartolome J, Bao J, Kasschau KD, Carrington JC, Voinnet O. 2006. Hierarchical action and inhibition of plant Dicer-like proteins in antiviral defense. Science 313: 68-71.

Dong Z, Han MH, Fedoroff N. 2008. The RNA-binding proteins HYL1 and SE promote accurate in vitro processing of pri-miRNA by DCL1. Proc Natl Acad Sci 105: 9970-9975.

Eamens AL, Blanchard CL, Dennis ES, Upadhyaya NM. 2004. A bidirectional gene trap construct suitable for T-DNA and Ds-mediated insertional mutagenesis in rice (Oryza sativa L.). Plant Biotechnol J 2: 367-380.

Fang Y, Spector DL. 2007. Identification of nuclear dicing bodies containing proteins for microRNA biogenesis in living Arabidopsis plants. Curr Biol 17: 818-823.

Förstemann K, Tomari Y, Du T, Vagin VV, Denli AM, Bratu DP, Klattenhoff C, Theurkauf WE, Zamore PD. 2005. Normal microRNA maturation and germ-line stem cell maintenance requires Loquacious, a double-stranded RNA-binding domain protein. PLoS Biol 3: e236. doi: 10.1371/journal.pbio.0030236.

Fujioka Y, Utsumi M, Ohba Y, Watanabe Y. 2007. Location of a possible miRNA processing site in $\mathrm{SmD} 3 / \mathrm{SmB}$ nuclear bodies in Arabidopsis. Plant Cell Physiol 48: 1243-1253.

Fusaro AF, Matthew L, Smith NA, Curtin SJ, Dedic-Hagan J, Ellacott GA, Watson JM, Wang M-B, Brosnan C, Carroll BJ, et al. 2006. RNAi-inducing hairpin RNAs in plants act through the viral defence pathway. EMBO Rep 7: 1168-1175.

Gasciolli V, Mallory AC, Bartel DP, Vaucheret H. 2005. Partially redundant functions of Arabidopsis DICER-like enzymes and a role for DCL4 in producing trans-acting siRNAs. Curr Biol 15: 14941500.

Gleave AP. 1992. A versatile binary vector system with a T-DNA organisational structure conducive to efficient integration of cloned DNA into the plant genome. Plant Mol Biol 20: 1203-1207.

Gregory RI, Chendrimada TC, Cooch N, Shiekhattar R. 2005. Human RISC couple microRNA biogenesis and post-transcriptional gene silencing. Cell 123: 631-640.

Han MH, Goud S, Song L, Fedoroff N. 2004. The Arabidopsis doublestranded RNA-binding protein HYL1 plays a role in microRNAmediated gene regulation. Proc Natl Acad Sci 101: 1093-1098.

Hellens RP, Edwards EA, Leyland NR, Bean S, Mullineaux PM. 2000. pGreen: A versatile and flexible binary Ti vector for Agrobacterium-mediated plant transformation. Plant Mol Biol 42: 819-832.

Hiraguri A, Itoh R, Kondo N, Nomura Y, Aizawa D, Murai Y, Koiwa H, Seki M, Shinozaki K, Fukuhara T. 2005. Specific interactions between Dicer-like proteins and HYL1/DRB-family dsRNA-binding proteins in Arabidopsis thaliana. Plant Mol Biol 57: $173-188$. 
Jiang F, Ye X, Liu X, Fincher L, McKearin D, Liu Q. 2005. Dicer-1 and R3D1-L catalyze microRNA maturation in Drosophila. Genes \& Dev 19: 1674-1679.

Jones L, Keining T, Eamens A, Vaistij FE. 2006. Virus-induced gene silencing of Argonaute genes in Nicotiana benthamiana demonstrates that extensive systemic silencing requires Argonautel-like and Argonaute4-like genes. Plant Physiol 141: 598-606.

Khvorova A, Reynolds A, Jayasena SD. 2003. Functional siRNAs and miRNAs exhibit strand bias. Cell 115: 209-216.

Kurihara Y, Watanabe Y. 2004. Arabidopsis mircoRNA biogenesis through Dicer-like 1 protein functions. Proc Natl Acad Sci 101: 12753-12758.

Kurihara Y, Takashi Y, Watanabe Y. 2006. The interaction between DCL1 and HYL1 is important for efficient and precise processing of pri-miRNA in plant microRNA biogenesis. RNA 12: 206-212.

Li RY, Qin Y, Macara BR, Cullen BR. 2003. Exportin-5 mediates the nuclear export of pre-microRNAs and short hairpin RNAs. Genes \& Dev 17: 3011-3016.

Liu Q, Rand TA, Kalidas S, Du F, Kim HE, Smith DP, Wang X. 2003. R2D2, a bridge between the initiation and effector steps of the Drosophila RNAi pathway. Science 301: 1921-1925.

Liu X, Park JK, Jiang F, Liu Y, McKearin D, Liu Q. 2007. Dicer-1, but not Loquacious, is critical for assembly of miRNA-induced silencing complexes. RNA 13: 2324-2329.

Mallory AC, Vaucheret H. 2006. Functions of microRNAs and related small RNAs in plants. Nat Genet 38: S31-S37.

Margis R, Fusaro AF, Smith NA, Curtin SJ, Watson JM, Finnegan EJ, Waterhouse PM. 2006. The evolution and diversification of Dicers in plants. FEBS Lett 580: 2442-2450.

Matranga C, Tomari Y, Shin C, Bartel DP, Zamore PD. 2005. Passenger-strand cleavage facilitates assembly of siRNA into Ago2-containing RNAi enzyme complexes. Cell 123: 607-620.

Mi S, Tao C, Hu Y, Chen Y, Hodges E, Ni F, Wu L, Li S, Zhou H, Long C, et al. 2008. Sorting of small RNAs into Arabidopsis Argonaute complexes is directed by the $5^{\prime}$ terminal nucleotide. Cell 133: 1-12.

Nakano M, Nobuta K, Vemaraju K, Tej SS, Skogen JW, Meyers BC. 2006. Plant MPSS databases: Signature-based transcriptional resources for analyses of mRNA and small RNA. Nucleic Acids Res 34: D731-D735.

Nakazawa Y, Hiraguri A, Moriyama H, Fukuhara T. 2007. The dsRNA-binding protein DRB4 interacts with the Dicer-like protein DCL4 in vivo and functions in the trans-acting siRNA pathway. Plant Mol Biol 63: 777-785.

Park W, Li J, Song R, Messing J, Chen X. 2002. CARPEL FACTORY, a Dicer homolog, and HEN1, a novel protein, act in microRNA metabolism in Arabidopsis thaliana. Curr Biol 12: 1484-1495.

Park MY, Wu G, Gonzalez-Sulser A, Vaucheret H, Poethig RS. 2005. Nuclear processing and export of microRNAs in Arabidopsis. Proc Natl Acad Sci 102: 3691-3696.

Pontier D, Yahubyan G, Vega D, Bulski A, Saez-Vasquez J, Hakimi MA, Lerbs-Mache S, Colot V, Lagrange T. 2005. Re- inforcement of silencing at transposons and highly repeated sequences requires the concerted action of two distinct RNA polymerases IV in Arabidopsis. Genes \& Dev 19: 2030-2040.

Rajagopalan R, Vaucheret H, Trejo J, Bartel DP. 2006. A diverse and evolutionarily fluid set of microRNAs in Arabidopsis thaliana. Genes \& Dev 20: 3407-3425.

Rand TA, Petersen S, Du F, Wang X. 2005. Argonaute2 cleaves the anti-guide strand of siRNA during RISC activation. Cell 123: 621629.

Reinhart BJ, Weinstein EG, Rhoades MW, Bartel B, Bartel DP. 2002. MicroRNAs in plants. Genes \& Dev 16: 1616-1626.

Saito K, Ishizuka A, Siomi H, Siomi MC. 2005. Processing of premicroRNAs by the Dicer-1-Loquacious complex in Drosophila cells. PLoS Biol 3: e325. doi: 10.1371/journal.pbio/0030235.

Schwarz DS, Hutvágner G, Du T, Xu Z, Aronin N, Zamore PD. 2003. Asymmetry in the assembly of the RNAi enzyme complex. Cell 115: 199-208.

Song L, Han M-H, Lesicka J, Fedoroff N. 2007. Arabidopsis primary microRNA processing proteins HYL1 and DCL1 define a nuclear body distinct from the Cajal body. Proc Natl Acad Sci 104: 54375442 .

Tagami Y, Inaba N, Kutsuna N, Kurihara Y, Watanabe Y. 2007. Specific enrichment of miRNAs in Arabidopsis thaliana infected with Tobacco mosaic virus. DNA Res 14: 1-6.

Takeda A, Iwasaki S, Watanabe T, Utsumi M, Watanabe Y. 2008. The mechanism selecting the guide strand from small RNA duplexes is different among Argonaute proteins. Plant Cell Physiol 49: 493500.

Tomari Y, Matranga C, Haley B, Martinez N, Zamore PD. 2004. A protein sensor for siRNA asymmetry. Science 306: 1377-1380.

Vaucheret H, Vazquez F, Crété P, Bartel DP. 2004. The action of ARGONAUTE1 in the miRNA pathway and its regulation by the miRNA pathway are crucial for plant development. Genes \& Dev 18: 1187-1197.

Vaucheret H, Mallory AC, Bartel DP. 2006. AGO1 homeostasis entails co-expression of miR168 and AGO1 and preferential stabilization of miR168 by AGO1. Mol Cell 22: 129-136.

Vazquez F, Gasciolli V, Crété P, Vaucheret H. 2004. The nuclear dsRNA binding protein HYL1 is required for microRNA accumulation and plant development, but not posttranscriptional transgene silencing. Curr Biol 14: 346-351.

Xie Z, Allen E, Wilken A, Carrington JC. 2005. DICER-LIKE4 functions in trans-acting small interfering RNA biogenesis and vegetative phase change in Arabidopsis thaliana. Proc Natl Acad Sci 102: 12984-12989.

Yang L, Liu Z, Lu F, Dong A, Huang H. 2006. SERRATE is a novel nuclear regulator in primary microRNA processing in Arabidopsis. Plant J 47: 841-850.

Zhu QH, Spriggs A, Matthew L, Fan L, Kennedy G, Gubler F, Helliwell C. 2008. A diverse set of microRNAs and microRNAlike small RNAs in developing rice grains. Genome Res 18: 14561465. 

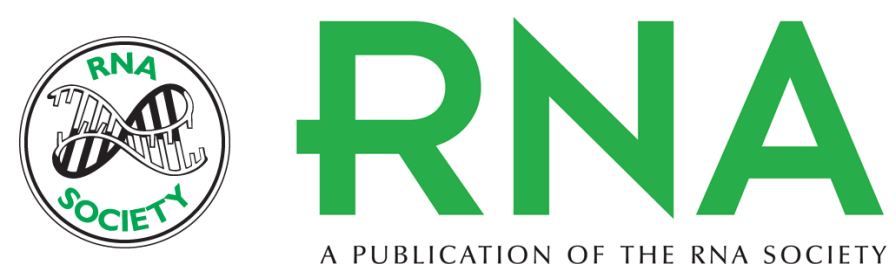

A PUBLICATION OF THE RNA SOCIETY

\section{The Arabidopsis thaliana double-stranded RNA binding protein DRB1 directs guide strand selection from microRNA duplexes}

Andrew L. Eamens, Neil A. Smith, Shaun J. Curtin, et al.

RNA 2009 15: 2219-2235 originally published online October 27, 2009

Access the most recent version at doi:10.1261/rna.1646909

Supplemental
Material http://rnajournal.cshlp.org/content/suppl/2009/10/02/rna.1646909.DC1

References This article cites 56 articles, 22 of which can be accessed free at:

http://rnajournal.cshlp.org/content/15/12/2219.full.html\#ref-list-1

License

Email Alerting Receive free email alerts when new articles cite this article - sign up in the box at the Service top right corner of the article or click here. 